\title{
Analysis of dispersion of heated effluent from power plant: a case study
}

\author{
VIKRAM SHAH $^{1}$, ANKIT DEKHATWALA $^{1}$, JYOTIRMAY BANERJEE $^{1, *}$ and A K PATRA ${ }^{2}$ \\ ${ }^{1}$ Department of Mechanical Engineering, Sardar Vallabhbhai National Institute of Technology, Surat 395007 , \\ India \\ ${ }^{2}$ Environment Survey Laboratory (ESL), Kakrapar Gujarat Site, Kakrapar, India \\ e-mail: jbaner@med.svnit.ac.in
}

MS received 18 May 2016; revised 17 November 2016; accepted 8 December 2016

\begin{abstract}
Thermal dispersion of heated effluent in a lake near nuclear power plant is analysed. Lake Bathymetry is established by data collection at a pre-planned matrix of sample points in the lake. Threedimensional geometric model of the lake is developed based on the geometric data collected, using a high accuracy GPS and a dead weight based depth meter at respective sample points of the lake matrix. A turbine type digital flow meter is used to measure the velocities at the intake and blow down points of the lake. Numerical analysis of flow and thermal dispersion is carried out using PLIC-VOF two-phase model with the two-equation $\mathrm{k}$-epsilon model for turbulence closure. Numerical results for varying flow and blow down temperature conditions and wind speed are studied. It is observed that the thermal gradients are steeper in the curved area near the blowdown point. Small increase in main inlet (inlet II) velocity suppresses the dispersion of high-temperature contours significantly. Thermal discharge and dispersed temperature is monitored using temperature sensor mounted floating buoy at various locations in the lake. It is established that the thermal dispersion is influenced by wind velocity and the presence of water hyacinth in the lake.
\end{abstract}

Keywords. Thermal dispersion; heated effluent; power plant.

\section{Introduction}

Water used in the power plant for cooling purposes is released as thermal effluent at a slightly elevated level of temperature to the nearby aquatic system. Nuclear power plants require $30-100 \%$ more cooling water than other types of plant with a comparable power output [1]. The mean water body temperature may not be significantly affected by the introduction of thermal effluent because of the large heat capacity of the waterbody. But there are possibilities of a steep rise in local temperature. The flora and fauna in the waterbody get affected by the steep local variations in temperature [2]. Increased local temperature has an impact on dissolved oxygen (DO) which in turn affect metabolism, reproduction, longevity and growth of inhibiting aquatic ecosystem [3]. Many times, the heated effluent leads to the elimination of weakly resistant species which are replaced by more tolerable, but nuisance ones. Examples of such species are Naegleria Fowleri (Braineating amoeba) [4] which has been reported to be harmful to humans as they enter and feed upon the brain tissues. The existence of such species near power plant discharge is due

*For correspondence to high local temperature which is favourable for their growth.

Most of the power plants are located near coastal areas, rivers, lakes or ponds and the thermal effluent from the power plants are released at an elevated temperature near the blowdown into this aquatic system. In order to address the issue of the aquatic health of the species near the blowdown, it is required to analyse the mechanism of dispersion of thermal effluent in the water body nearfield of the blowdown and to establish a zone of influence. Analysis of thermal dispersion is complex due to variation in physical properties of a water body due to the presence of weeds, water hyacinth and other aquatic species, the complexity of flow domain, variation of wind speed above the waterbody, thermal radiation from the water surface and other meteorological factors.

Literature reports analysis of thermal dispersion in different aquatic environments using analytical, numerical and experimental methods. El-Alfy [5] analytically established a relationship between the excess temperature and plume area/flow rate for Talkha and El-Kurimat power station in Egypt. The analytical results were found to follow trends similar to those reported by field measurements. However, the quantitative estimation from analytical results differed significantly from the measured 
field data. This was due to the one-dimensional approximation of the analytical model so proposed. Rosen et al [6] used Plane Projection (PP) of wastewater thermal pollutant vector on Jiu River to estimate thermal discharge area. The effluent discharged water mass flow rate and the temperature was used to obtain the isotherms at the surface of the water in terms of complex geometrical shapes. For simplified approximations, the geometrical shape reduced to an elliptical distribution of surface isotherm contours. Entesar and El-Ghorab [7] physically modelled a portion of Nile river at north Giza power plant by scale ratio of 1:50 and tried to evaluate the mixing zone. They reported that mixing zone depends on the flow regime, the effluent discharge and the excess temperature above the ambient at the outfall. The results showed that the mixing zone area increases with increasing effluents and decreases with increasing flow in the river.

Literature in this area reported the development of a numerical model for thermal dispersion in the water body near the power plant exhaust [8-13]. Chieh [8], Zacharias and Ferentino [9] and Yamanaka et al [10] developed 2D model to predict thermal dispersion numerically. Zeng et al [11] and Fan et al [12] developed 3D numerical model considering unsteady turbulent flow and heat transport. However, no comparative assessments with experimental field measurements or model studies were reported by these researchers.

Literature also reports the use of commercial soft tools for simulation of thermal dispersion in lakes, rivers and coastal environments [14-26]. Abbaspour et al [14], Lowe et al [15], Liu et al [16] and Ahmed et al [17] used MIKE software in order to estimate thermal dispersion in coastal and confined water bodies. MIKE requires bathymetric data, stationary tidal variation, wind speed input and water discharge temperature to numerically estimate thermal dispersion. Demenet and Bae [18] and Prodanovic [19] used TELEMAC-3D to model the red sea and Lake Ontario respectively. Demenet and Bae [18] reported physical modelling of the red sea by scale ratio of 1:43. But their numerical results were not in close agreement with the results obtained in the physical model study. This was due to large scale ratio used by them. Dargahi and Shimelis [20] used Generalized Environmental Modelling System for Surface waters (GEMSS) to investigate hydrodynamic characteristics of lake Tana. Sun et al [21] used 3D model COHERENS for thermal dispersion analysis in Bohai sea. Yasser et al [22] used Delft3D for analysis of thermal effluent mixing in Suez Gulf. Mukhtasor et al [23] developed 2D hydrodynamics module for thermal dispersion analysis using Resources Management Associates (RMA2

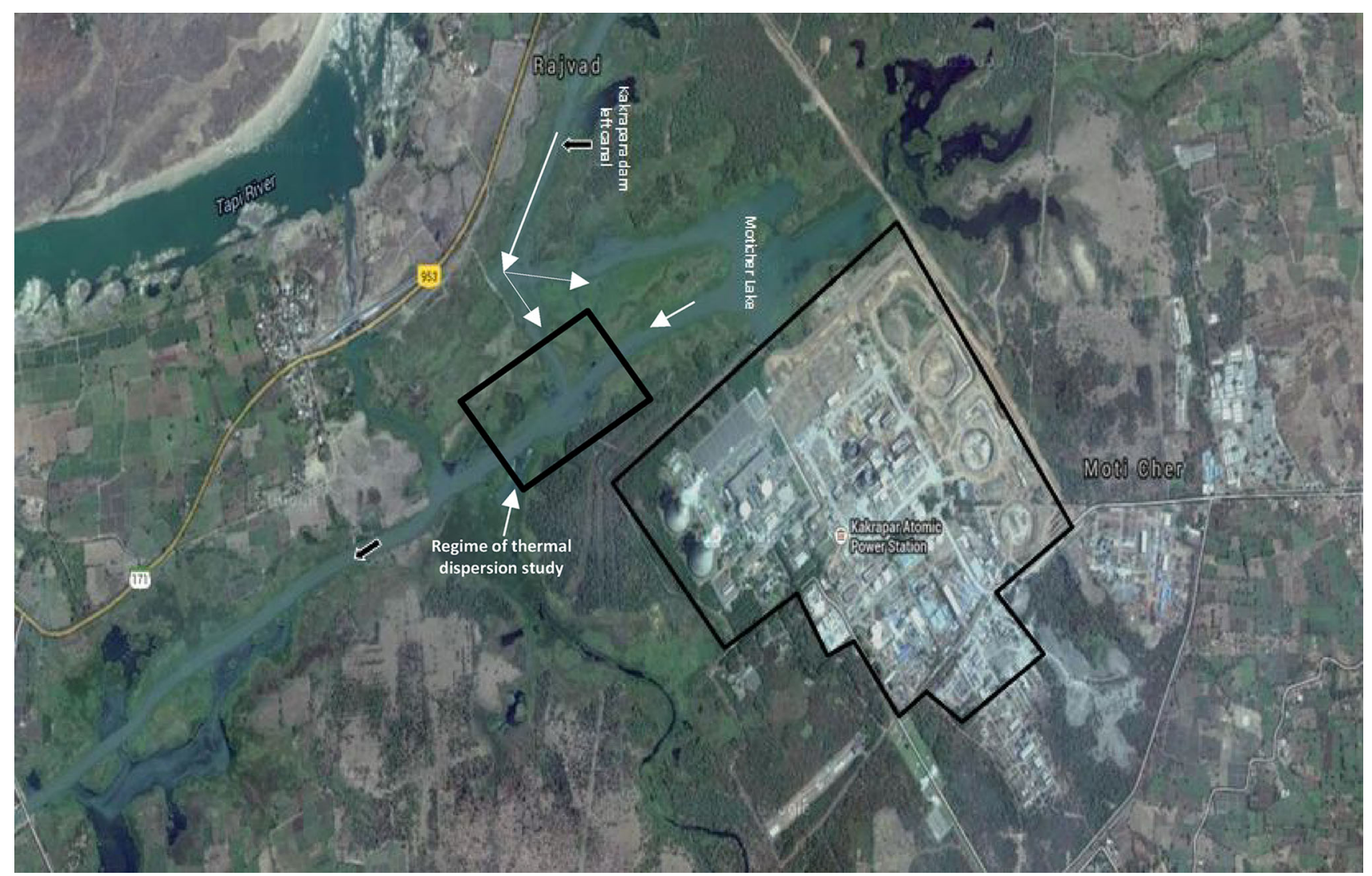

Figure 1. Aerial view of Moticher Lake and Kakrapara Atomic Power Plant, Gujarat. 


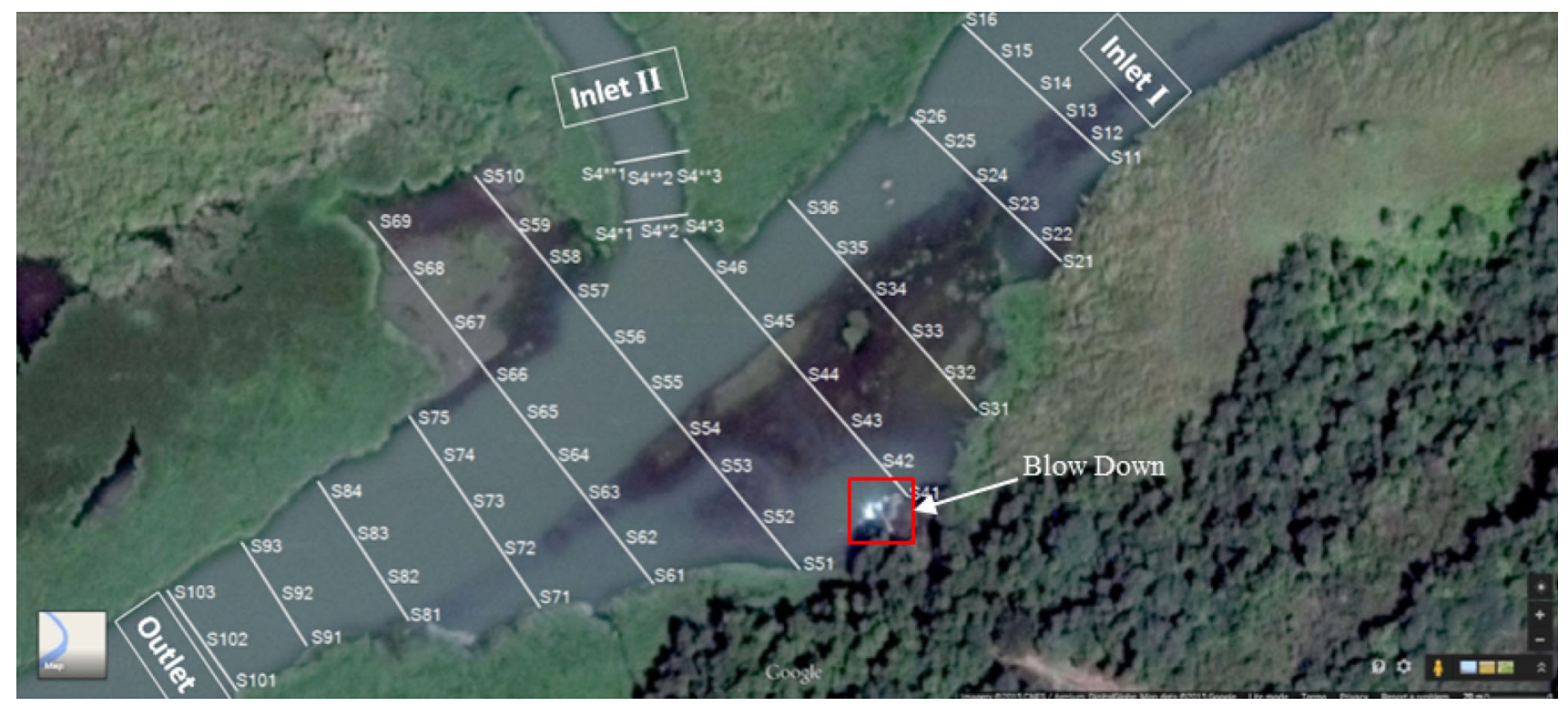

Figure 2. Matrix of sample points considered for collection of bathymetric data.

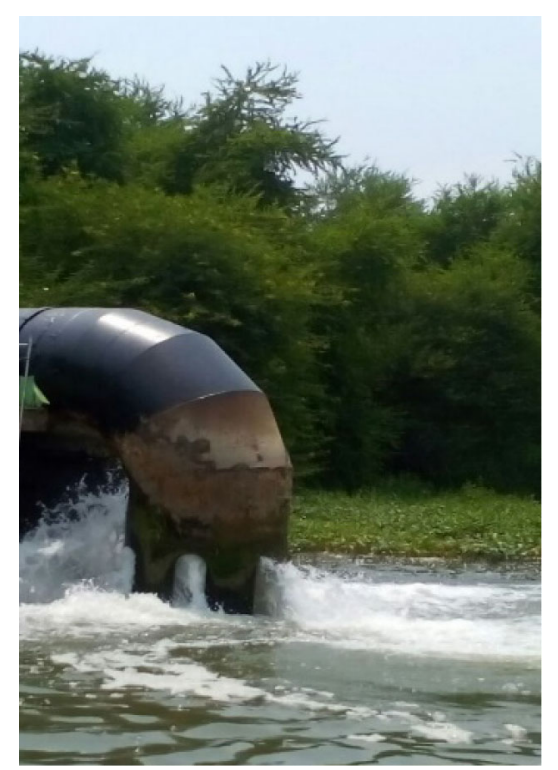

(a)

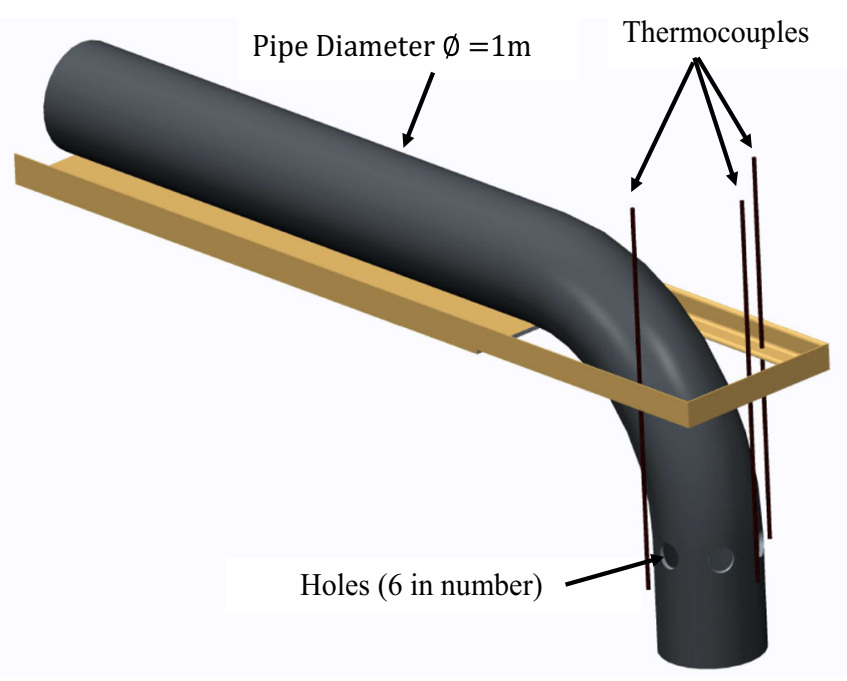

(b)

Figure 3. (a) Blowdown pipe showing the peripheral holes. (b) Attachment used for inserting the temperature probes near blowdown.

and RMA4), a finite element method. Fossati et al [24] used finite element model RMA-10 to model thermal dispersion in Montevideo bay. German et al [25] used finite element method for the numerical simulation of the dispersion of the thermal outflow in Huelva estuary. The numerical simulations carried out using soft tools have mostly been used by the researchers to demonstrate the mechanism of dispersion only.
Wind speed and direction are the dominant factors to drive the isotherm patterns in the aquatic system. A stronger wind would make the water temperature mix more in the vertical direction. Some authors have accounted for the wind speed over the water body for accurate thermal analysis. Sasmal et al [26] used three-dimensional (3D) Princeton Ocean Model (POM) with a constant momentum flux of reversing wind speeds of 2 and $10 \mathrm{~m} / \mathrm{s}$. A wider 
spread of the plume was demonstrated in their work for low wind speeds. As the wind speed tends to increase, the directional spread of plume dispersion was shown to become narrow.

Present work is a case study of numerical analysis and experimental field measurement for analysing the dispersion of thermal effluent released into the waterbody of Moticher lake from the operating units of Kakrapar Atomic Power Plant (KAPS). KAPS is a nuclear power plant located on the southern bank of Moticher Lake, in the downstream of Kakrapar weir on Tapi River. KAPS currently has a running capacity of $440 \mathrm{MW}(2 \times 220 \mathrm{MW})$ with a future expansion of $1400 \mathrm{MW}(2 \times 700 \mathrm{MW})$ in the installation at the same premises. Surface discharge method is adopted for an average volume flow rate of $5000 \mathrm{~m}^{3} / \mathrm{h}$ of effluent being released into the Moticher Lake [27]. Taking into consideration the up gradation of existing power generation capacity, the wastewaters discharged in the same lake could represent notable environmental impact, concerning aquatic ecosystems in the lake. In order to evaluate the need for controlling the temperature of water discharged from the power plant, the lake water temperature distribution near the blow down was proposed to be examined. Dispersion of thermal effluent released near the blowdown is analysed and reported in the present work.

Most of the literature demonstrated numerical or analytical prediction of thermal dispersion. However, there is very few literature available on field measurements and model analysis. The present paper reports field measurements carried out in the lake using specially designed experimental buoy for local temperature measurement at different position and depth of the lake. A model laboratory scale experimental test facility is developed for validation of the numerical model used in the present work. The
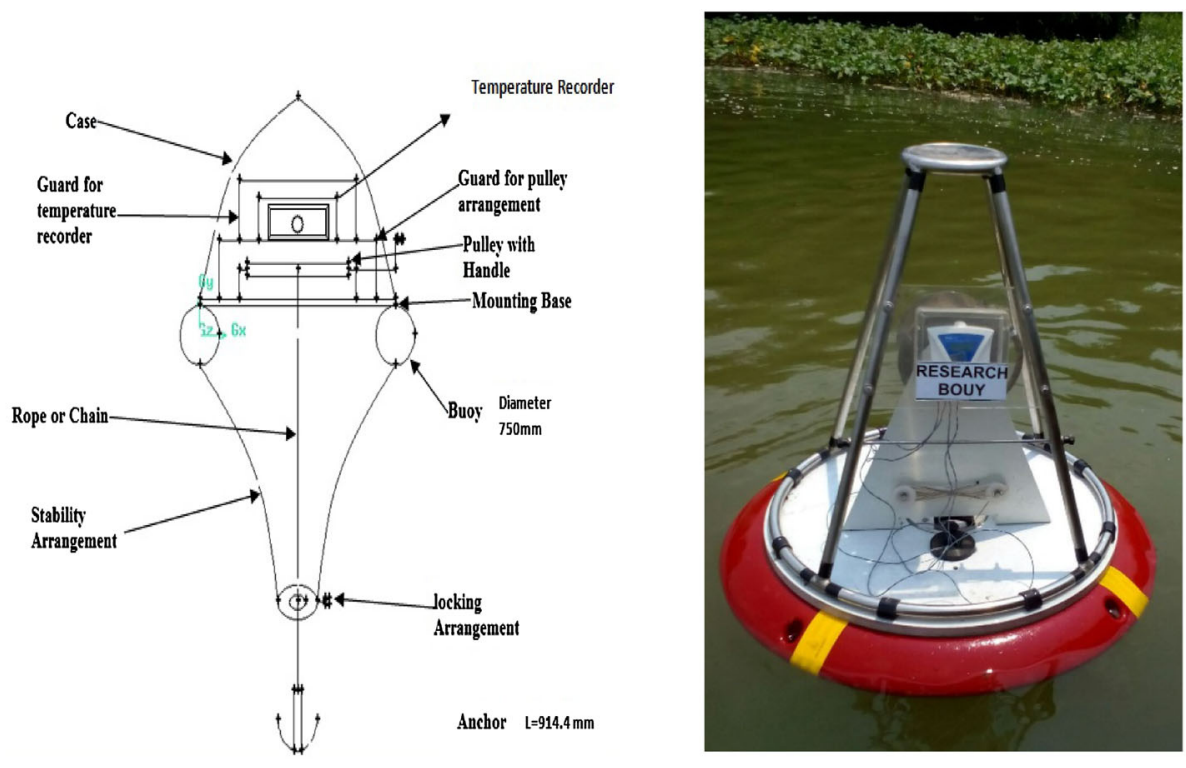

(a)

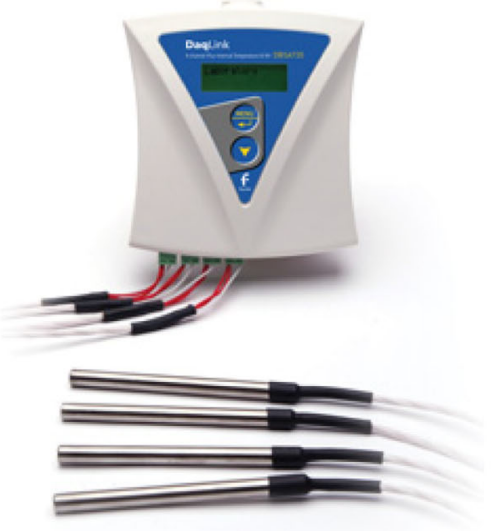

(b)

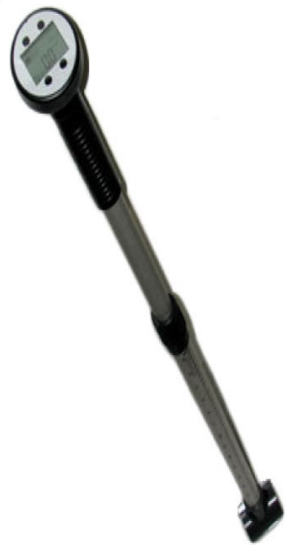

(c)

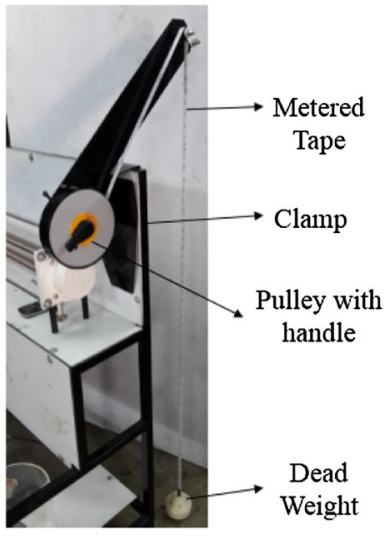

(d)

Figure 4. (a) Components of Buoy and its installation in Moticher Lake, (b) temperature sensors (PT-100) with multi input Data logger (Daqlink), (c) digital turbine-type flow probe for velocity measurement and (d) depth meter. 


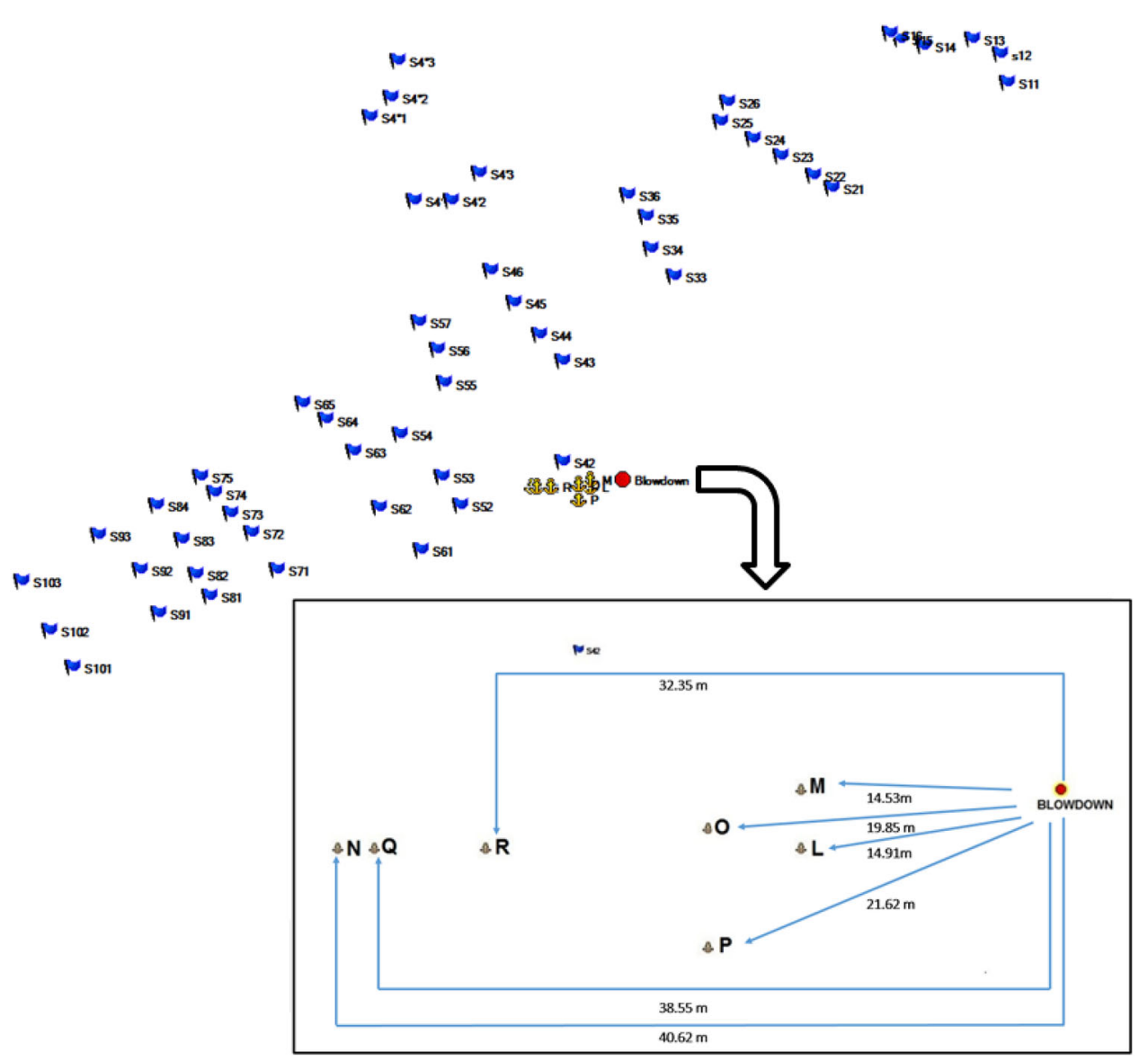

Figure 5. Location of buoy on the lake for continuous monitoring of temperature and different depth.

Table 1. Matereological data for different days of data collection during September 2015 and Januray 2016.

\begin{tabular}{lccccc}
\hline Sampling date & $\begin{array}{c}\text { Average air } \\
\text { temperature }\left({ }^{\circ} \mathrm{C}\right)\end{array}$ & $\begin{array}{c}\text { Average wind } \\
\text { speed }(\mathrm{m} / \mathrm{s})\end{array}$ & $\begin{array}{c}\text { Blowdown mass flow } \\
\text { rate }\left(\mathrm{m}^{3} / \mathrm{h}\right)\end{array}$ & $\begin{array}{c}\text { Average ambient water } \\
\text { temperature }\left({ }^{\circ} \mathrm{C}\right)\end{array}$ & $\begin{array}{c}\text { Weather } \\
\text { condition }\end{array}$ \\
\hline $11 / 09 / 2015$ & 35.1 & 1.3 & 2600 & 31 & Sunny day \\
$12 / 10 / 2015$ & 34.0 & 0.6 & 2500 & 29.5 & Cloudy day \\
$28 / 10 / 2015$ & 33.0 & 1.5 & 2500 & 29 & Sunny day \\
$24 / 11 / 2015$ & 28.6 & 1.5 & 6200 & 26 & Cloudy day \\
$25 / 11 / 2015$ & 25.1 & 1.7 & 2500 & 25 & Cloudy \\
$06 / 01 / 2016$ & 26 & 1.4 & 2500 & & Sunny day \\
$08 / 01 / 2016-12 /$ & 27 & 1.5 & & & Sunny day \\
$01 / 2016$ & & & &
\end{tabular}

validated numerical method is then used for numerical prediction of thermal dispersion in the lake.

\section{Overview of the lake}

Moticher Lake is the heat sink for the Kakrapara Atomic Power Plant. Source of water for cooling is Kakrapar Left Dam Canal located on Tapi River which supplies around1200 cusec of water [27]. This flow from Kakrapar Left Dam Canal gets segregated into two parts, one to the intake region and other to the blowdown of the Plant as shown in figure 1 . The plant is currently operating with two reactors of $220 \mathrm{MW}$ capacity each. The blowdown of heated effluent is not directly released into the mainstream flow of the lake. Blowdown pipe releases the effluent in an extended section of the lake on the left bank as shown in figure 2. Also, the discharge from the blowdown pipe is not 


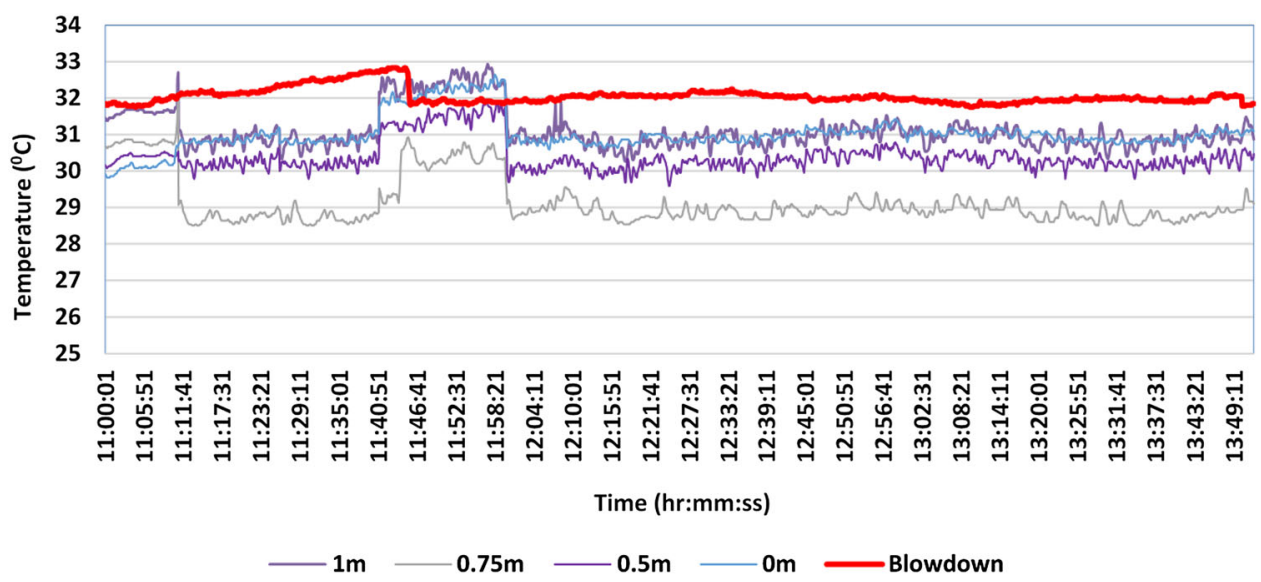

Figure 6. Timeline of temperature data recorded by data logger along depth at point M on a winter day 6th January 2016.

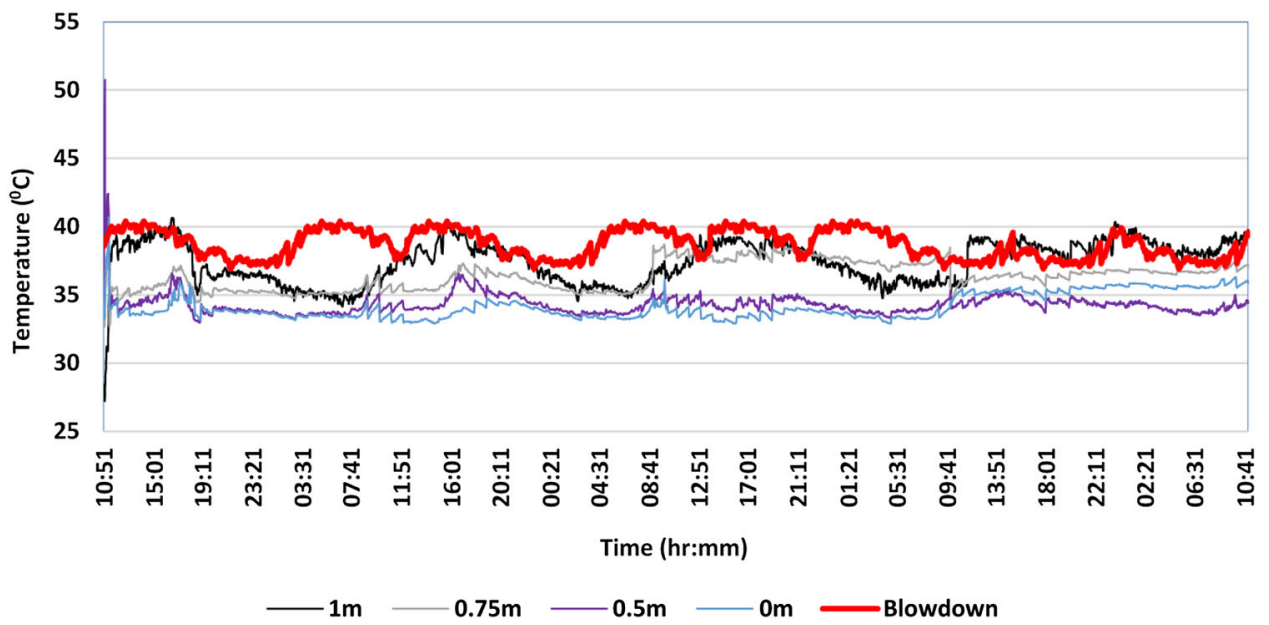

Figure 7. Timeline of temperature data recorded by data logger along depth at point P on 11th September 2015.

released only to the depth of the lake. There are holes made on the surface of the blowdown pipe which allows the heated effluent to be released at the surface level also.

\subsection{Generation of Lake Bathymetry}

Bathymetry is required for geometric modelling of the lake, more importantly, because the depth of the lake is observed to vary significantly. Dost and Mannaerts [28] first described the bathymetric generation of lake ecosystem using GPS, portable sonars, remote sensing data and GIS software. In a similar line, the bathymetry is developed for the Motichar lake in the mainstream lake near the blowdown. Lake domain shown in figure 2 is considered for the analysis of thermal dispersion. There are two ambient water inflow routes, a blowdown effluent release and a single outlet. InletI is for the flow of unutilized water from the intake of the plant. Due to large pressure drop in the passage through intake area of the plant, Inlet-I has considerably low velocity as compared to Inlet-II which supplies the mainstream flow into the lake. The Effluent is released from blow down through the circular pipe of $1 \mathrm{~m}$ diameter. To create the geometric model, a pre-planned matrix of sample points across the lake shown in figure 2 is chosen to measure the depth of the lake at these points. A Motorboat carrying GPS receiver with a special in-house designed depth meter is used to record the depth, latitude and longitude at the matrix points. GPS readings and depth for respective sample points were used to generate the 3D lake geometry. It is established from the depth measurements that the minimum depth of the lake near the blowdown is of the order of $4 \mathrm{~m}$ while the maximum depth in the lake domain where bathymetry is generated is of the order of $15 \mathrm{~m}$.

\section{Experimental monitoring of thermal dispersion in lake}

For the experimental analysis of thermal dispersion, the temperature is monitored near the blowdown at different distances along the lake and at different depths of the lake. 
Figure 3(a) shows the blowdown of the Power plant. The heated effluent is discharged through the holes on the periphery of $1 \mathrm{~m}$ diameter blowdown pipe and is also discharged into the bulk of the lake through the exit of the blowdown pipe.

\subsection{Instruments used for temperature monitoring}

The temperature of water at blowdown is measured using three temperature sensors (Resistance temperature detectors), PT-100 with a sensitivity of $0.01^{\circ} \mathrm{C}$. A special structure is fabricated as shown in figure $3(\mathrm{~b})$ to hold these sensors at the blowdown for effluent temperature monitoring. A battery operated data logger is used to store data from the temperature sensors. The data logger can store continuous temperature data detected by the sensors installed near blowdown pipe at a time interval of $\Delta t=5 \mathrm{~s}$. The blowdown temperature is estimated as the average of the temperature sensed by these three temperature detectors.

An in-house buoy which floats in the waterbody, shown in figure 4(a) is designed and fabricated in order to facilitate the collection of temperature data from different distances in the lake and at a different depth near the blowdown. The buoy consists of a buoy ring, anchor, and rope-pulley with locking mechanism. This buoy can be anchored at any location in the lake. The anchor with locking arrangement ensures that the buoy is not carried away with the flow and ensures data collection at a defined position at different depths of the water level. Multi-input data logger as shown in figure 4(b) with PT-100 long wired sensors are installed in the buoy at different depths. The data loggers at blowdown and the buoy stores temperature date simultaneously from the sensors. Digital Turbine-type flow probe is used to measure the velocity at various points in the Lake. The average velocity of the inlet and blowdown are measured using turbine type digital flow probe shown in figure 4(c). The in-house designed depth meter used for Bathymetry is shown in figure 4(d).

\subsection{Data collection}

Figure 5 shows the position and shortest distance between the buoy and blowdown where data collection was facilitated on some typical days in the point matrix of the lake. The buoy is kept at different sample locations as indicated in figure 5. Meteorological data for typical seven different sampling periods during September 2015 to January 2016 is tabulated in table 1.

The simultaneously measured temperature data from the blowdown and the buoy is collected on different days. The high temperature difference between the blowdown and ambient water is observed majorly due to plant shut-down, full load operating conditions of the plant, high ambient temperature during summer or low water level in the Moticher Lake. Two different sets of data are presented in

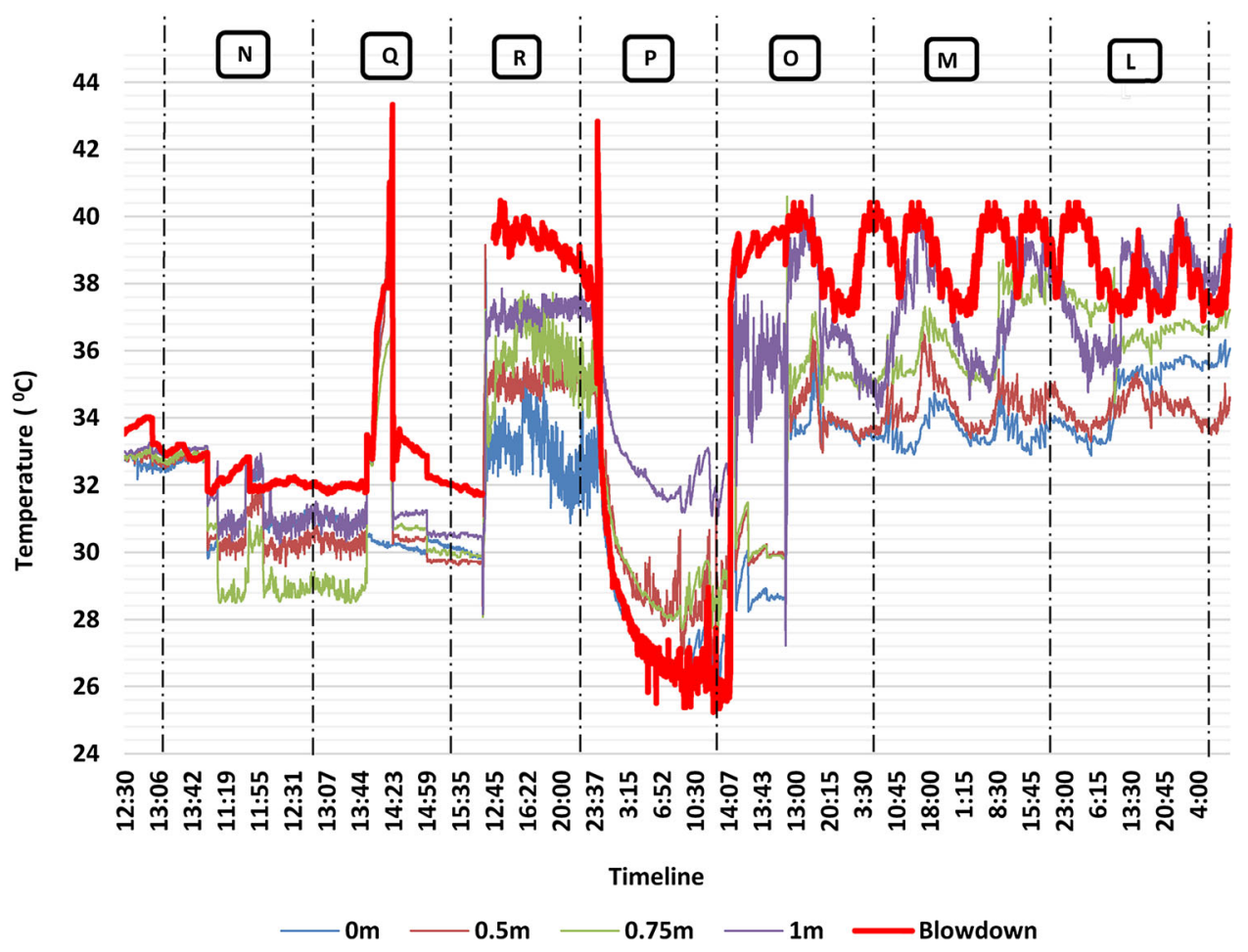

Figure 8. Timeline of temperature data recorded by data logger at different depth and at different points of the lake on 8th--12th January 2016. 
figures 6 and 7. Figure 6 shows the data collected at the blowdown and at different depths at point $\mathrm{M}$ in the lake on a winter day of 6th January 2015. The temperature data is stored at every $5 \mathrm{~s}$ time interval. Figure 6 shows a data set where for a part of the time period of release, the blowdown discharge temperature increases with time. This is followed by a sudden drop in discharge temperature and the discharge temperature remains almost constant thereafter. The sudden change in blowdown temperature is sensed over a period of time at all depths with a time delay as depicted in figure 6. It is observed that the temperature from the blowdown discharge cools down by $2-5^{\circ} \mathrm{C}$ by the time it reaches the buoy.

Figure 7 shows the data collected at the blowdown at different depths at point $\mathrm{P}$ on 11th September 2015. Figure 7 shows a data set where the blow down discharge shows a near sinusoidal variation. Red line indicates the blowdown temperature while other lines show the temperature variation at different positions of the sensors attached to the buoy at a depth of $0,0.5,0.75$ and $1 \mathrm{~m}$ respectively. The sinusoidal signal from the discharge is sensed by the temperature sensors at point $M$ with a varying magnitude at different depth and with a time delay. From the collected data at point $\mathrm{M}$ in figure 8 a difference of $3-8^{\circ} \mathrm{C}$ is observed between blowdown discharge and water temperature at a different depth of the lake.

Figure 8 shows continuously monitored data of temperature at the blowdown and different positions of the lake at a different depth measured during 8-12th January 2016. It is established from the measured data that the effluent discharge temperature is typically maintained below $40^{\circ} \mathrm{C}$ as is specified by a regulatory authority of Gujarat. The effluent temperature is regulated by the plant before the discharge is released into the blowdown.

\subsection{Estimation of zone of influence based on field measurement}

Environmental protectionists report the concept of 'mixing zone' as the region within which a contaminant plume is legally allowed to interact with the surrounding water. The 'contaminant' may be heat, a chemical or any other quantity that presents a possible impact to the environment. The mixing zone should fulfil the following conditions (Entesar and Ghorab [7]):

1. The mixing zone shall be limited to an area or volume as small as possible to minimise adverse impacts on aquatic life and other beneficial uses.

2. The mixing zone shall not impact or cause the migration of fish or other aquatic life. There shall be a safe and adequate passage for living organisms with no deleterious effects on their populations.

In the present work, the temperature date monitored at different locations for different blowdown temperature is used to generate a zone of influence on the surface of lake water due to the thermal effluent release. The zone of influence is estimated in the present work as the area on the lake water surface till which the temperature is $2^{\circ} \mathrm{C}$ above the ambient lake temperature (International regulatory authorities suggest $3^{\circ} \mathrm{F}$ as permissible limit [27]). Based on the field data analysis it is found that the temperature measured by the temperature sensors located at point $\mathrm{N}$ and Q (figure 5) shows a negligible difference with the ambient water temperature. Only at fewer occasions, the temperature sensed at point $\mathrm{R}$ (figure 5) exceeded $2^{\circ} \mathrm{C}$ above the ambient temperature. The zone of influence established based on the field data is compared with the numerically estimated zone of influence and is discussed in the subsequent sections of this paper. The analysis is carried out on laboratory scale model for validation of the numerical method used for the present work.

\section{Numerical and experimental analysis on laboratory scale model}

A laboratory scaled model is developed for validation of the numerical method used for the dispersion analysis. Necessary similarity laws were maintained for appropriate

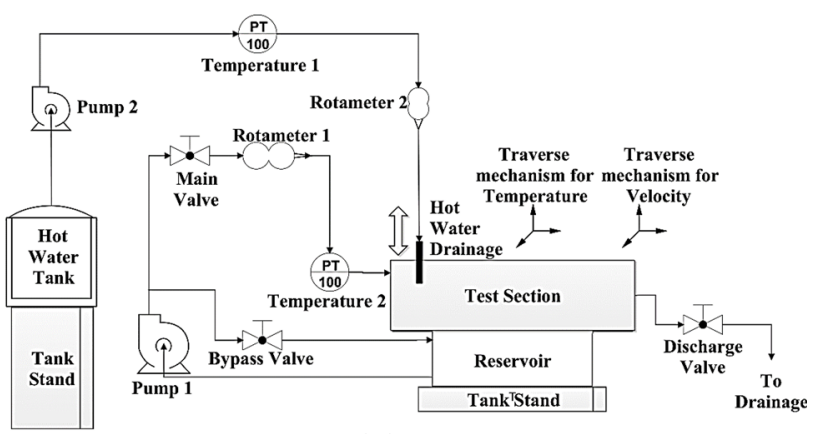

(a)

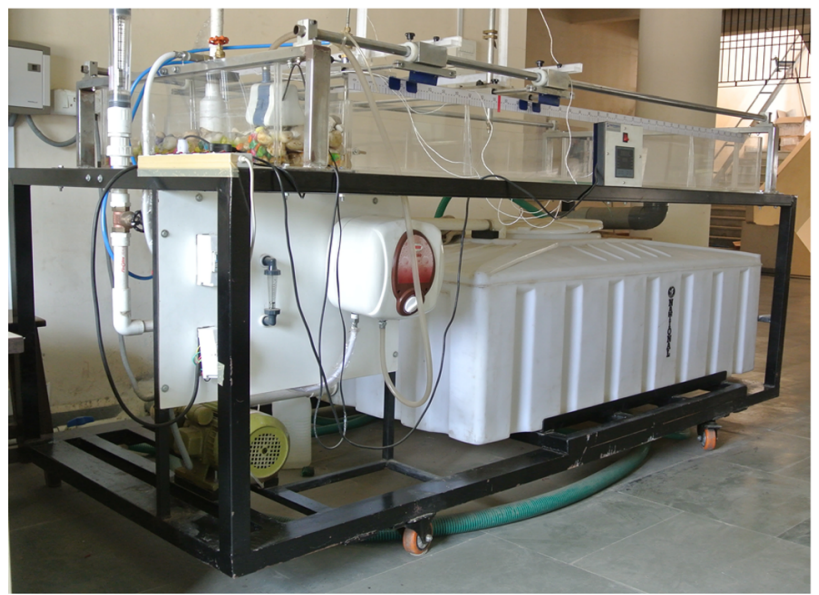

(b)

Figure 9. (a) Schematic layout and (b) photograph of the laboratory scale test facility developed for thermal dispersion analysis. 
model scaling. The criteria used include limits of Reynolds number value for ensuring fully turbulent flows in the model. Reynolds number of heated effluent discharge in the model, $\mathrm{Re}_{e}$, is kept higher than 2000 and Reynolds number $\mathrm{Re}_{a}$, for free surface flows is kept larger than 500. The characteristic dimension used for estimation of Reynolds number of effluent discharge is the diameter of the effluent discharge pipe while for free surface flow the depth of water is used as the characteristic dimension. Another requirement in the full-field model is similarity of ambient flow patterns which is matched by equality of Densimetric Froude number (considering buoyancy) between model and prototype, expressed as:

$$
\frac{U_{m}}{\sqrt{g H_{m} \frac{\Delta \rho_{m}}{\rho_{m}}}}=\frac{U_{p}}{\sqrt{g H_{p} \frac{\Delta \rho_{p}}{\rho_{p}}}}
$$

Here, $H$ is water depth; $U$ is water velocity and $g$ is the acceleration of gravity. Densimetric Froude number is maintained below 0.6 in the present model. Modelling of convective spread requires that, in addition to Eq. (1), the
Densimetric Reynolds number of the model exceeds a certain limiting value, which is satisfied by assuring that the convective heat spread is all limited in the model area. Densimetric parameters are essential for scaling in the present work because the effluent discharge in the Kakrapar Atomic Power Plant (KAPS) is partly at the surface level and partly till a depth of water. This balance in surface discharge and depth-wise discharge allows for reducing the zone of influence. In KAPS a part of the thermal effluent is released at the surface level through holes created on the pipe wall while a fraction of the heated effluent is pushed into the bulk of fluid by the mass flow rate of discharge. This fraction of the fluid mass is subjected to a buoyant force due to the difference in density associated with the temperature gradient.

Figure 9(a) shows the schematic layout of thermal dispersion test setup consisting of four major components: hot water unit, ambient water unit, and test section and temperature measurement unit. The photograph of the developed test facility for model scale thermal dispersion analysis is shown in figure 9(b). Continuous water supply is

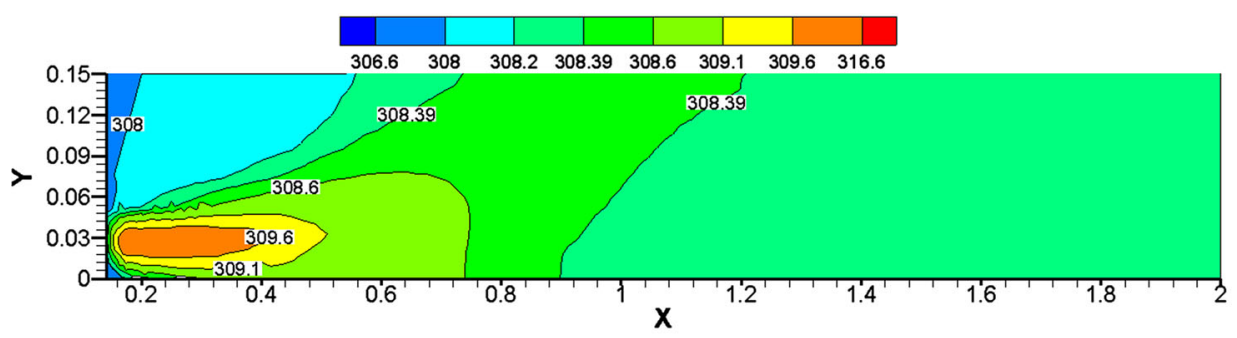

(a)

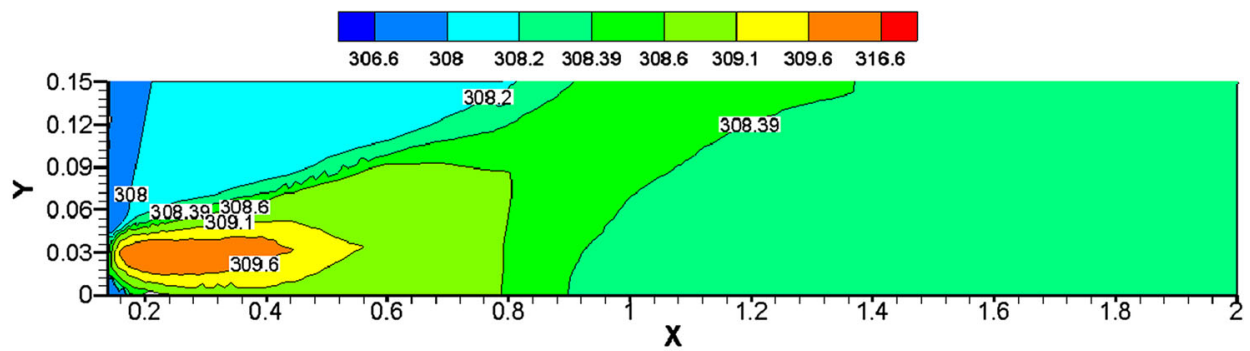

(b)

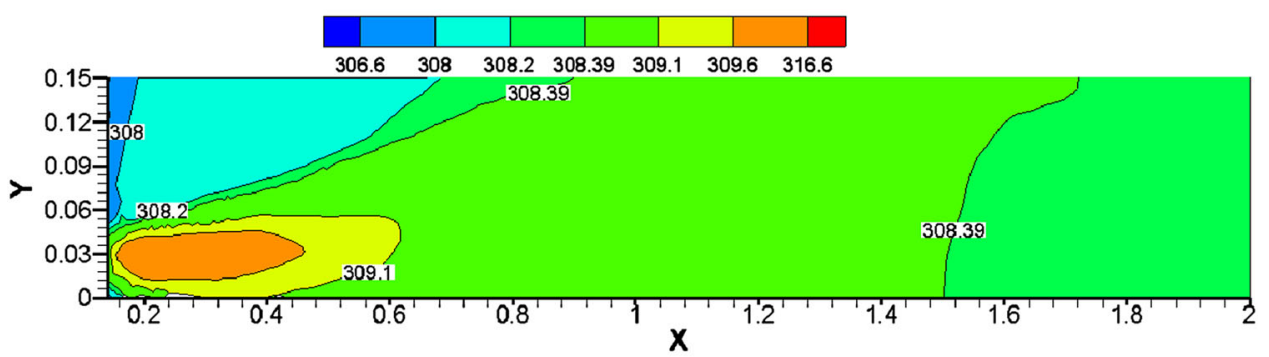

(c)

Figure 10. Temperature contours (in K) established from numerical simulation for air velocity (a) $v=0 \mathrm{~m} / \mathrm{s},(\mathbf{b}) v=0.2 \mathrm{~m} / \mathrm{s}$ and $(\mathbf{c}) v=$ $0.3 \mathrm{~m} / \mathrm{s}$. 


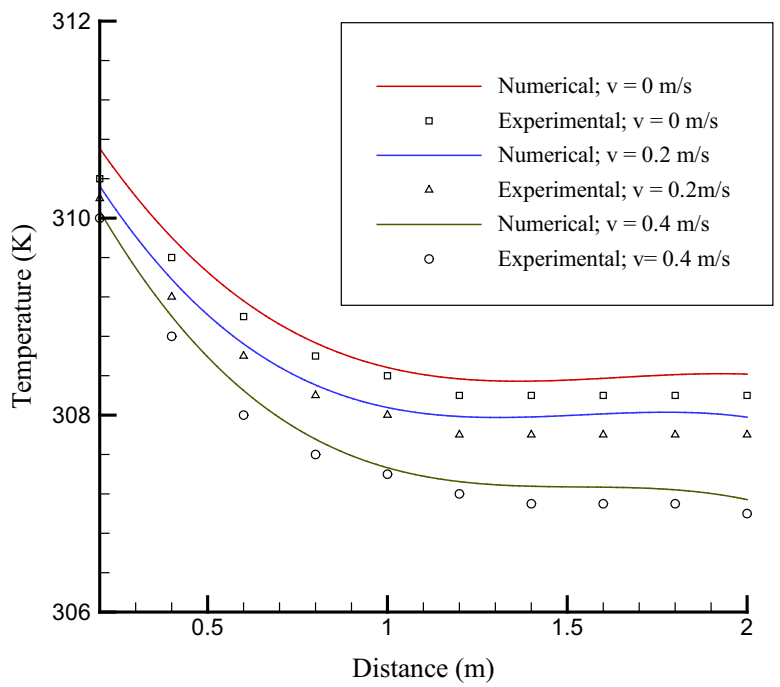

(a)

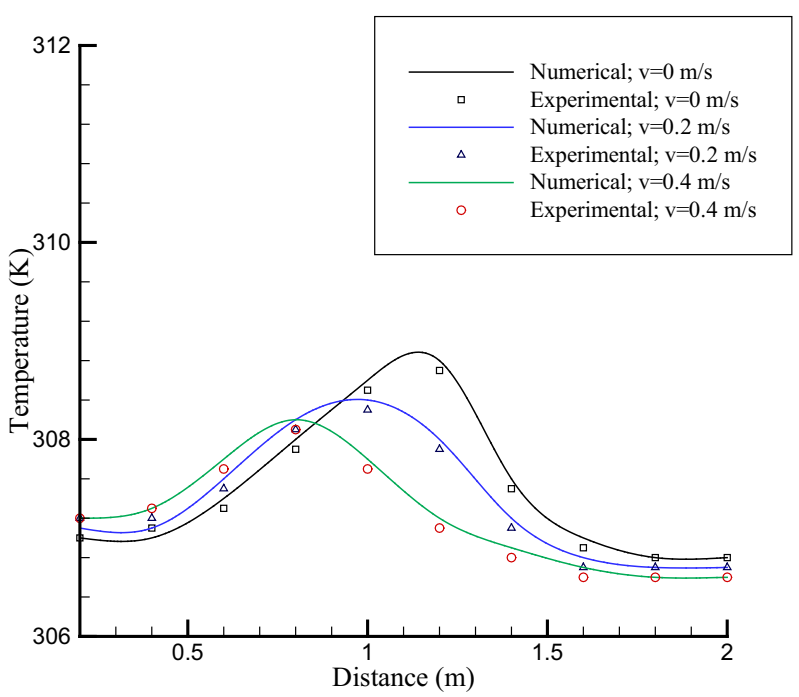

(b)

Figure. 11. Comparison on temperature (in $\mathrm{K}$ ) variation measured experimentally with those obtained numerically along the centre line of the domain (a) at a height of $0.03 \mathrm{~m}$ above ground level (b) at water free surface level for different air velocities.

provided in the test section from a 10001 reservoir using a centrifugal pump. A glove valve is used for controlling the water flow rate along with a bypass valve. The water flow rate is measured using a rotameter. A bypass valve is incorporated between the main supply line and the reservoir to remove excess water back to the reservoir. Hot water is supplied from the constant temperature hot bath by a centrifugal pump into the effluent discharge flow line. The hot water bath temperature is controlled by a rheostat and is capable of supplying constant temperature hot water in the range of $300-320 \mathrm{~K}$. The hot effluent discharge (jet diameter $1 \mathrm{~cm}$ ) is maintained by a glove valve and the flow rate is measured using rotameter. Resistance Temperature
Detector (PT-100) sensors are used for temperature measurement and Acoustic Doppler Velocimetry (ADV) is used for velocity measurement in the test section. These sensors are mounted on a traverse system which can be moved along any plane in the test section. A data logger is installed for storage and indication of input signals from the sensors.

\subsection{Validation of numerical model}

The flow and thermal dispersion in the test section is numerically modelled using the dimensions of the model experimental setup. The modelled domain is $2 \mathrm{~m}$ long, $0.88 \mathrm{~m}$ wide and $0.17 \mathrm{~m}$ deep. PLIC-VOF model is used for estimation of an interface between the air and water and standard k- $\varepsilon$ model is used for turbulence modelling. The numerical results obtained for water flow rate of $600 \mathrm{LPH}$, effluent flow rate of $150 \mathrm{LPH}$ and the temperature difference of $10 \mathrm{~K}$ are shown in figure 10 . Ambient water temperature condition is $306.6 \mathrm{~K}$. Boussinesq approximation [26] is used for buoyancy induced flow. Hot flow is released from the effluent opening.

Figure 10(a) shows the temperature contours when the air above the ambient water is assumed to be at standstill. The effluent plume rises upward to the water surface in far field due to density difference. Figure 10(b) and (c) shows the temperature contours when air flows with different velocities in the direction of mainstream water flow. Due to air motion, the advection of temperature contours in the longitudinal direction is more pronounced. The temperature contours are dispersed more when the velocity of air is increased from $0.2 \mathrm{~m} / \mathrm{s}$ to $0.3 \mathrm{~m} / \mathrm{s}$. The temperature profile is measured by RTD sensors (PT-100) mounted on traverse system in the test setup shown in figure 11 . Constant air flow over water in the test section is provided using a blower. Air flow rate is monitored using an anemometer.

Figure 11 shows a comparison of experimentally measured temperature variation along the length of main flow with those obtained numerically at two different depths in the test section for cases with and without air flow. The increase in air velocity causes faster spreading of the plume in the downstream direction. Though the temperature values are slightly over predicted in the numerical model, the experimental results are comparable with numerically estimated values. The differences are attributable to the approximations associated with the numerical model. The numerical model over predicts the temperature.

Figure 12 shows a comparison of surface temperature distribution obtained from the numerical simulation to those obtained from the measurements near the water surface. Solid lines represent isotherms obtained from numerical predictions while the dashed lines represent the isotherms constructed based on measurement of temperature at the water surface level. It can be inferred from the surface measurement that the released heated effluent 


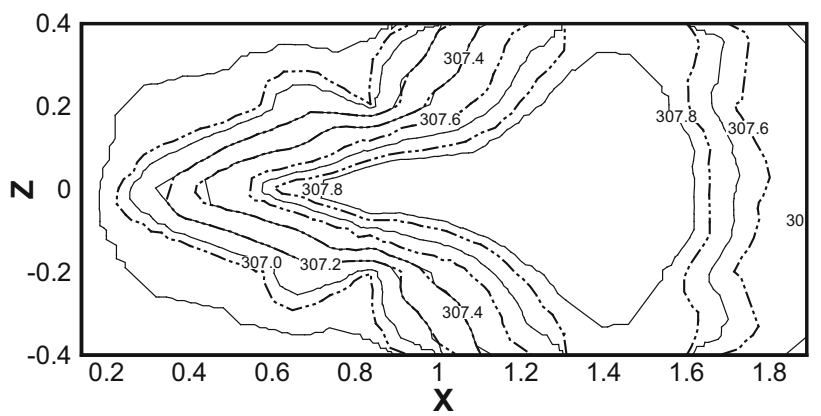

Figure 12. Comparison of numerically obtained surface temperature contours with the measured temperature near the water surface.

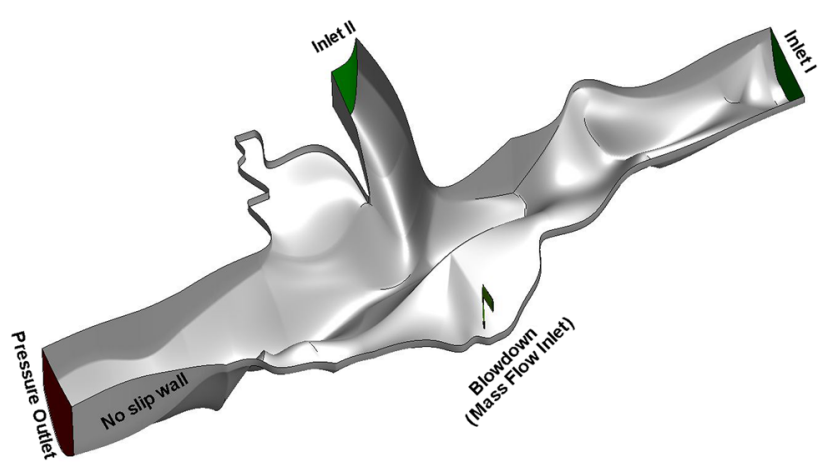

Figure 13. Three dimensional geometric model and boundary conditions.

initially rises upward due to its lower density and is then dispersed mostly near the surface. This was also demonstrated in the numerical results shown in figure 11.

\section{Numerical analysis of thermal dispersion in lake}

Numerical simulations are carried out for estimation of temperature distribution in the lake for varying inlet and blowdown flow rates and different blowdown temperatures. Since the depth of the lake varies significantly from $4 \mathrm{~m}$ near the blowdown to $15 \mathrm{~m}$ in the mainstream lake, the bathymetry of the lake is used to develop the geometric model of the lake domain and the numerical results are generated considering the variation of depth in the domain. Results of numerical simulations for boundary conditions obtained from field measurements are discussed in this section.

\subsection{Numerical model for the lake with boundary conditions}

Geometric model of the flow domain generated for analysis is shown in figure 13. The thermal effluent discharge pipe bends $2 \mathrm{~m}$ downward to meet the lake surface. The depth of the lake just below the blowdown in significantly low. In order to balance the hot effluent dispersion along the surface and along the depth of the river, the effluent is released from the periphery of the pipe through six holes as shown in figure 4(a) in addition to the main exit from the pipe into the water. This type of arrangement causes effluent to discharge in all directions at the surface of the lake and reduces the depth-wise penetration. A part of the water is released at the surface level through these holes while rest of the water moves along the depth by gravity.

Bottom of the domain and side of the domain are subjected to no-slip boundary conditions at ambient

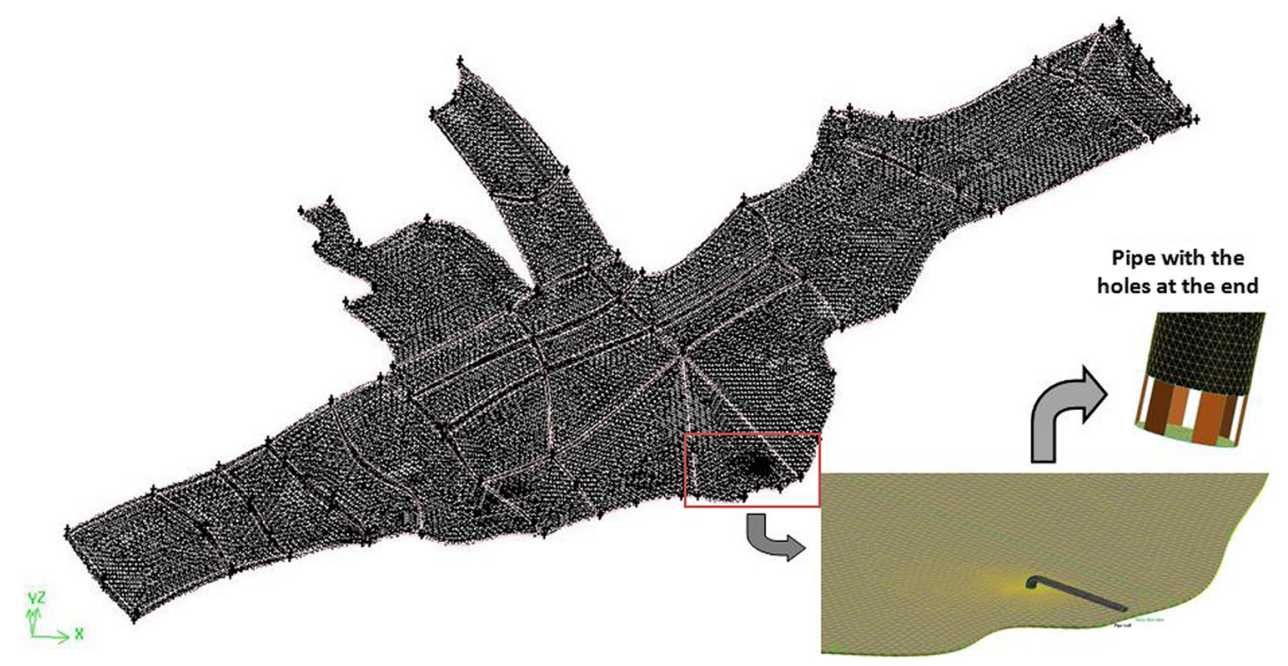

Figure 14. Geometric domain of the lake and pipe with unstructured mesh. 


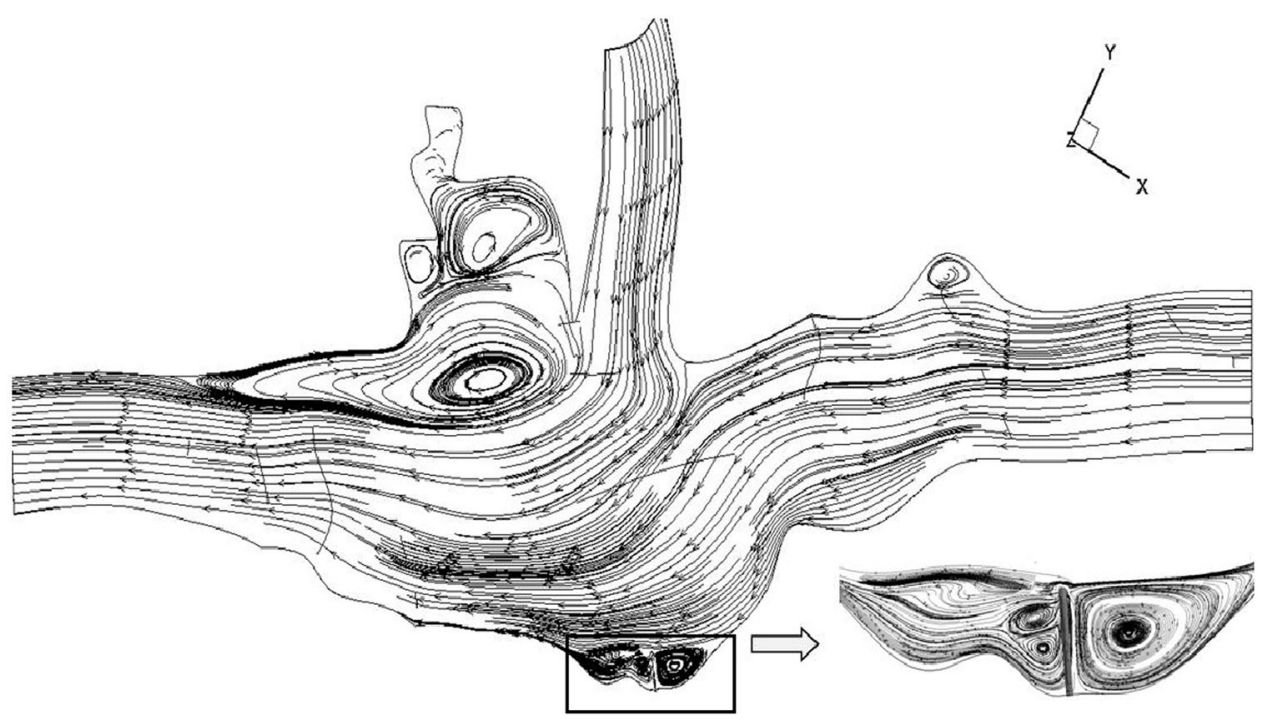

(a)

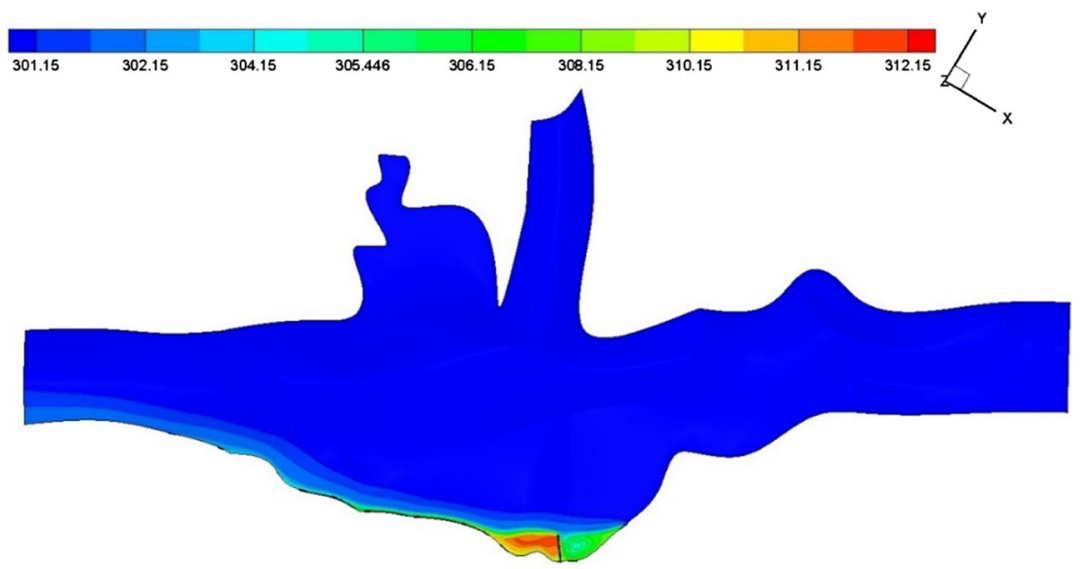

(b)

Figure 15. (a) Streamlines showing flow structure near the blow down and (b) temperature contours for blow down temperature of $12 \mathrm{~K}$ above ambient water temperature and blowdown mass flow rate of $2400 \mathrm{~m}^{3} / \mathrm{h}$.

temperature. Velocity inlet boundary conditions are defined at inlet-I and inlet-II. Effluent is released at a temperature higher than ambient temperature. The exit of flow is subjected to pressure outlet boundary conditions. Entire domain is initialized at ambient flow temperature. Heat transfer due to solar radiation is also considered on the lake surface for single phase calculations without wind flow. Theoretical Maximum radiation is assumed to fall with solar ray tracing computation method. North and East orientation of domain is specified properly. Starting time is given at afternoon 4 P.M. on 15th of the June. The solar load was updated at every 10-time step.

Since a part of the effluent from the blowdown pipe is released to the surrounding, PLIC-VOF based two-phase air-water model is used. The lake domain is meshed with unstructured triangular elements as shown in figure 14 with fine mesh near the blowdown. Six equal-spaced holes at the periphery of blowdown pipe end are specified as shown in figure 3 through which a part of the effluent flows. Mass flow rate is defined at a pipe section above the holes and interface boundary conditions are assigned at the equalspaced holes on the pipe periphery.

\subsection{Numerical analysis}

The time averaged measured velocities from field measurement are provided as input to the numerical model. Results shown in figure 15 are for Inlet I and Inlet II specified with average velocities of $0.6 \mathrm{~m} / \mathrm{s}$ and $1.25 \mathrm{~m} / \mathrm{s}$ respectively. Streamlines shown in figure 15(a) demonstrate the vortex structure near the blowdown. Two opposing vortex structures are observed near the blowdown due to effluent flow, the shape of the lake and inlet flow 


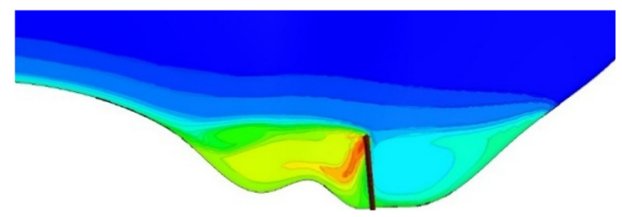

$301.15 \quad 302.15303 .15304 .15305 .15306 .15 \quad 307.15 \quad 308.15309 .15 \quad 310.15311 .15312 .15$

(a)

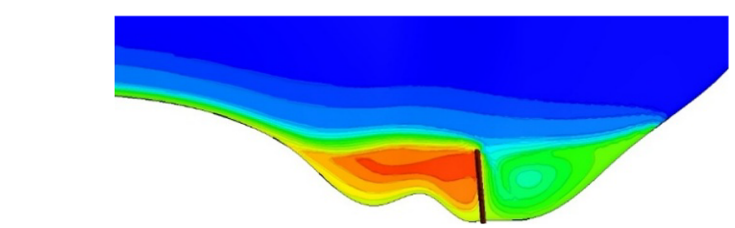

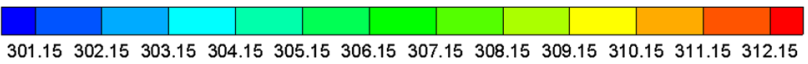

(b)

Figure 16. Temperature distribution demonstrating the rise of buoyant plume (a) at depth $0.5 \mathrm{~m}$ from free surface (b) at free surface.

conditions. The flow is streamlined along the outlet in rest of the domain. A large vortex is formed as flow from inlet II gets orthogonally diverted towards the outlet and a small set of vortices near blowdown are formed due to concavity of lake near the blowdown.

Vortex structure far away from the blowdown does not get affected by the elevated temperature distribution as shown in figure 15(b). The downstream of the blowdown is at higher temperature and the presence of high temperature contours are observed mostly near the left bank of the lake. Hence the left bank of the lake is susceptible to create thermal shocks for aquatic species. This may affect the organisms present near the left bank.

Figure 16 shows the influence of rise of the buoyant plume on the temperature distribution at different depth of water near the blowdown. At a depth of $0.5 \mathrm{~m}$ from the free surface a clear structure of the streak of thermal plume is visible in figure 16(a). As the plume rises it gets dispersed. At the free surface as shown in figure 16(b) the temperature gets diffused to a larger domain downstream of the plume.

Figure 17(a) shows water temperature distribution perpendicular to the direction of flow at three traverse sections: $7 \mathrm{~m}$ upstream, at blowdown and $7 \mathrm{~m}$ downstream. The temperature drops to ambient within $10 \mathrm{~m}$ from the blowdown. Line passing through the blowdown pipe centre has the maximum value of peak temperature difference as shown in figure 17(a). The temperature distribution at $7 \mathrm{~m}$ upstream in traverse direction shows that the surface temperature rises after some distance and then it drops. This depicts that the thermal plume released along with the depth at blowdown undergoes buoyancy force due to temperature difference and moves upward at a distance away from the pipe location. This point in the surface shows an elevation of temperature. The vortex centre at the upstream of blowdown has a relatively lower temperature, however vortex centres on the downstream shows relatively higher temperature due to the influence of buoyant plume.

Figure 17(b) shows water temperature distribution along the direction of flow at three longitudinal sections in the zone of influence. The temperature profile in a longitudinal direction parallel to the main flow along three different planes at $4 \mathrm{~m}, 8 \mathrm{~m}$ and $10 \mathrm{~m}$ away from the blowdown is shown. The results show an increase in temperature at some distance downstream of blowdown due to buoyant plume rising at that level. A drop of temperature is observed at the centre of the upstream vortex demonstrated in figure 17(b). This is due to circumferential advection of temperature by the vortex.

5.2a Influence of flow rate from blowdown and inlet-II: The thermal dispersion pattern gets altered due to change in flow conditions. Effect of variation in inlet velocity, blow down mass flow rate and blow down temperature difference on thermal dispersion are analysed and reported here. Blowdown mass flow rate depends on the volume of water taken from the intake. The measured flow rates released by the plant at the blowdown reported in table 1 shows values ranging from $2500 \mathrm{~m}^{3} / \mathrm{h}$ to $6200 \mathrm{~m}^{3} / \mathrm{h}$ on different days for which data is reported in table 1 . Plant running at full load consumes more water for cooling. The variation of temperature distribution near the blowdown at the free surface level when the blowdown flow rate is doubled from $2400 \mathrm{~m}^{3} / \mathrm{h}$ to $4800 \mathrm{~m}^{3} / \mathrm{h}$ in shown in figure 18 . As the mass flow rate of blowdown increases, high temperature contours spread more in the downstream direction. However the high temperature contours remain confined near the left bank because of advection of ambient water from inlet-II. As a result, the zone of influence is not significantly altered in the direction normal to mainstream flow as shown in figure 18. Inlet II is the main source of ambient water. Dispersion of thermal effluent is greatly affected by the flow of inlet II. Figure 19 shows the variation of temperature distribution at a free surface level near the blowdown when the average velocity from Inlet II is doubled. It is observed that, increase in inlet II velocity leads to more dilution and more dispersion of thermal effluent and leads to smaller area of influence as shown in figure 19(b). At lower from velocity from inlet II, thermal effluent spreads more towards the left bank and the zone of influence is larger.

$5.2 \mathrm{~b}$ Influence of blowdown temperature: The water release from Kakrapar dam to the Moticher Lake is controlled by the irrigation department. When there is an inadequate availability of water in the lake premises (generally observed during summer season) the effluents are released at higher temperatures with low mass flow rate at blowdown exit. Figure 20 shows the temperature distribution at the free surface level when the blowdown temperature is doubled. The large temperature difference 


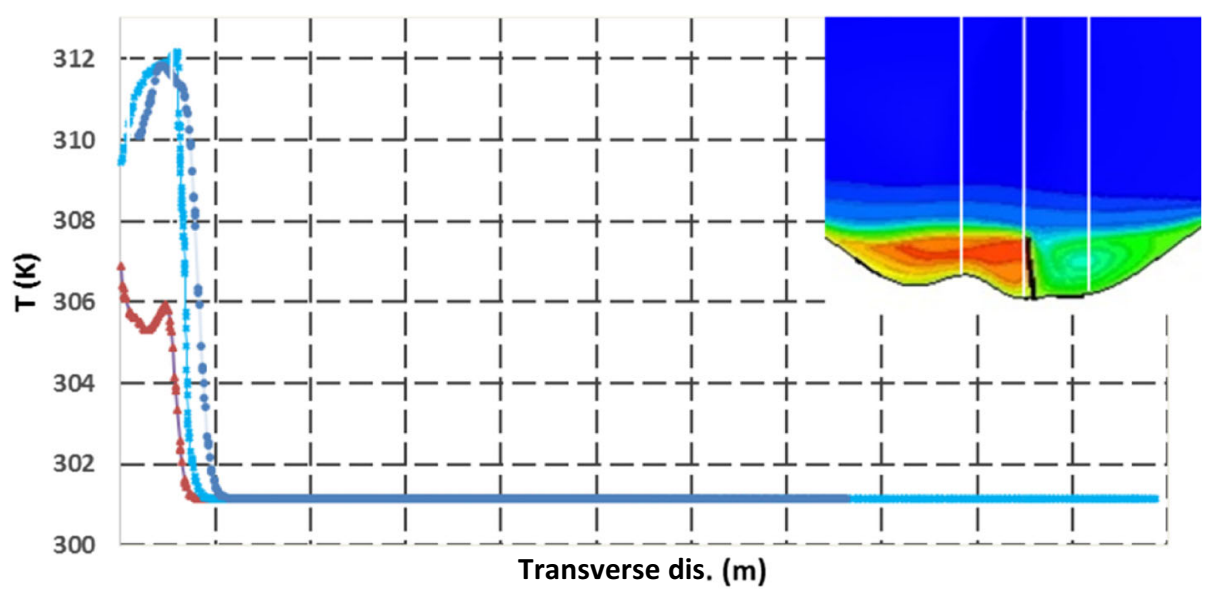

$\multimap 7 \mathrm{~m} \mathrm{U} / \mathrm{S}$ of blowdown $\ldots$ at blowdown $\ldots 7 \mathrm{~m} \mathrm{D} / \mathrm{S}$ of blowdown

(a)

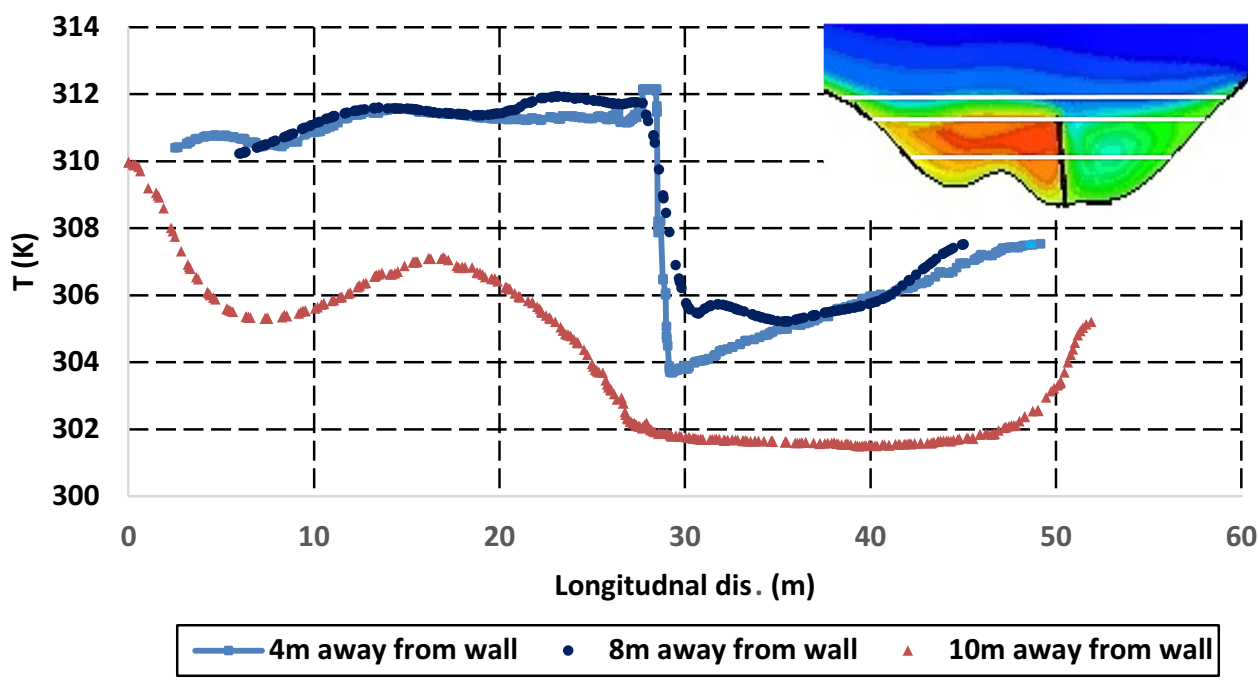

(b)

Figure 17. (a) Transverse profile of temperature distribution. (b) Longitudinal profile of the temperature distribution.

between ambient and blowdown causes rise in local temperature near blow down as shown in figure 20. The local temperature rise observed is however not associated with major enlargement of the zone of influence. This is due to strong influence of ambient flow from inlet-II.

5.2c Influence of wind speed: As per the wind velocity data available from the meteorological department, the wind speed over the water surface reaches to a high value of $3 \mathrm{~m} / \mathrm{s}$ at times and the direction of the wind is mostly opposite to the main flow of effluent from the blowdown (North-West). Figure 21 shows the result of numerical simulation obtained using two-phase VOF model considering air velocity of $3 \mathrm{~m} / \mathrm{s}$. The temperature contours in figure 21 shows broadening of plume in the direction of air flow.

\subsection{Zone of influence}

The zone of influence in the lake domain is highly localized near the blowdown. Location of blowdown opposite to inlet (from inlet-II) water flow pushes the released thermal effluent from the blowdown pipe to the concave region near blowdown and the zone of influence remain near the downstream left bank of the lake. For all realistic conditions of blowdown heat release, inlet water flow and wind conditions, the thermal dispersion is limited to the region shown in figure 22 as per the present numerical simulations.

The zone of influence obtained from the numerical simulations are compared to those estimated based on experimental measurements of the lake in figure 23. The comparison is made for numerical results with wind flow. 

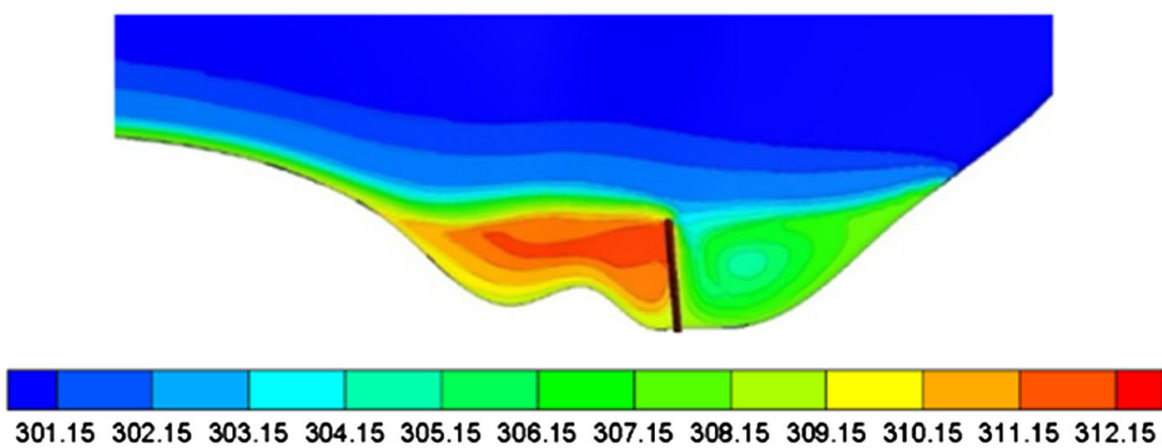

(a)
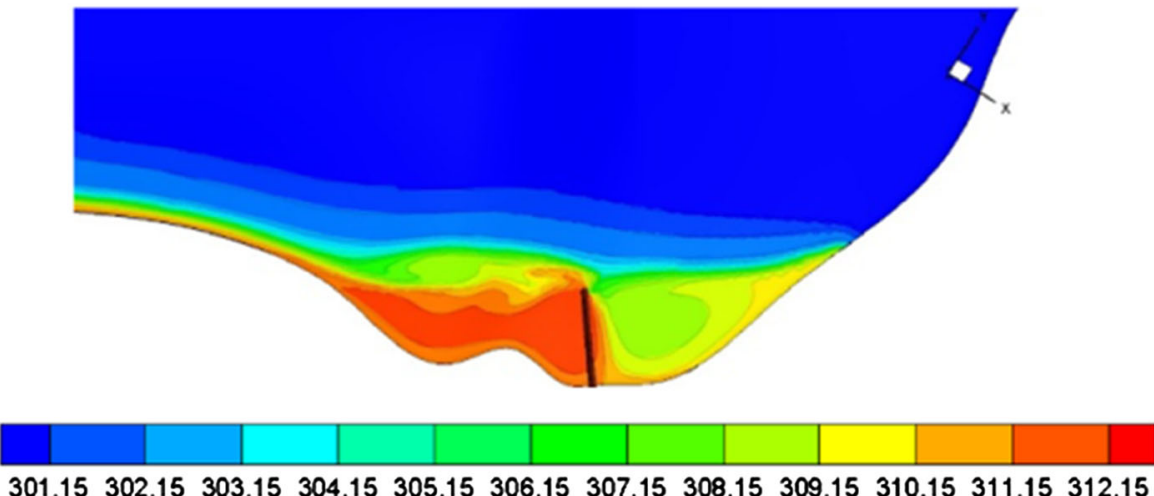

(b)

Figure 18. Influence of blowdown mass flow rate on thermal dispersion (a) $2400 \mathrm{~m}^{3} / \mathrm{h}$ and (b) $4800 \mathrm{~m}^{3} / \mathrm{h}$.

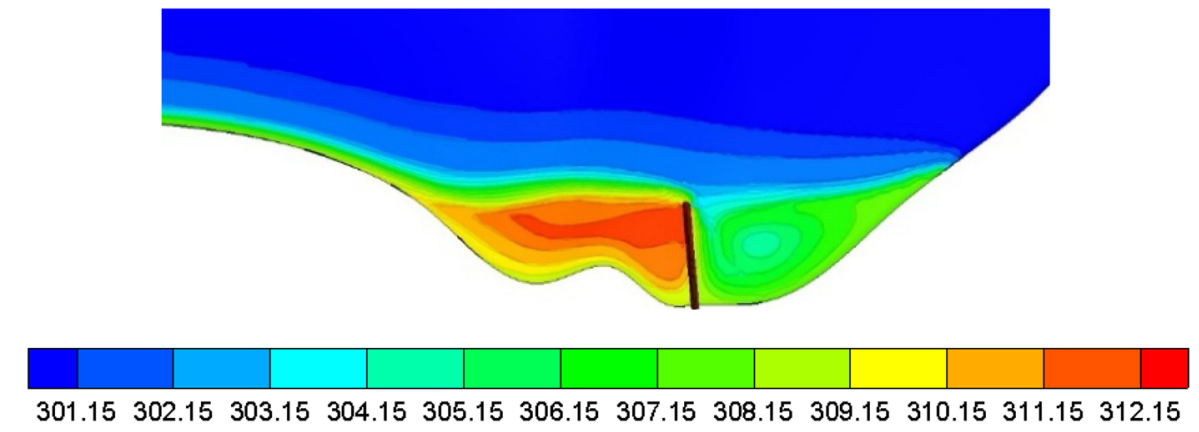

(a)
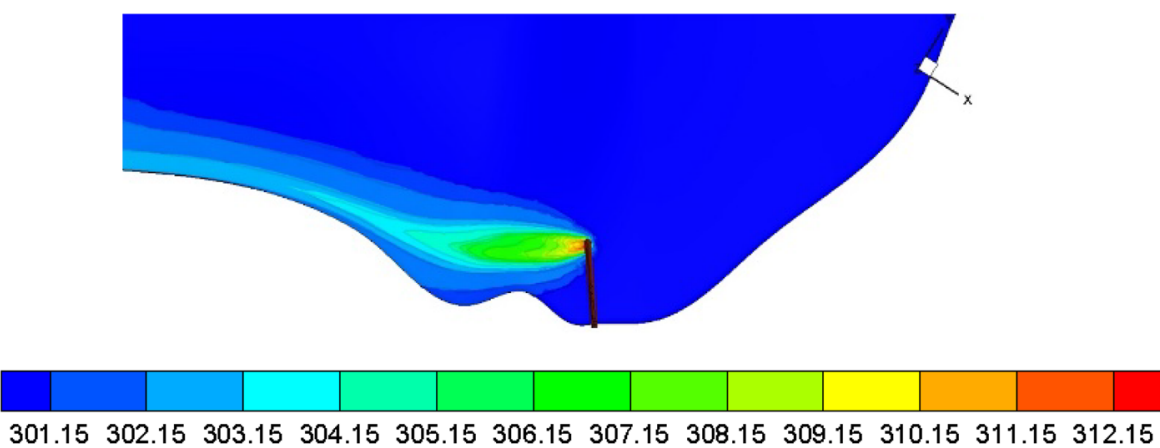

(b)

Figure 19. Effect of velocity of inlet II on thermal dispersion (a) $0.8 \mathrm{~m} / \mathrm{s}$ and (b) $1.6 \mathrm{~m} / \mathrm{s}$. 


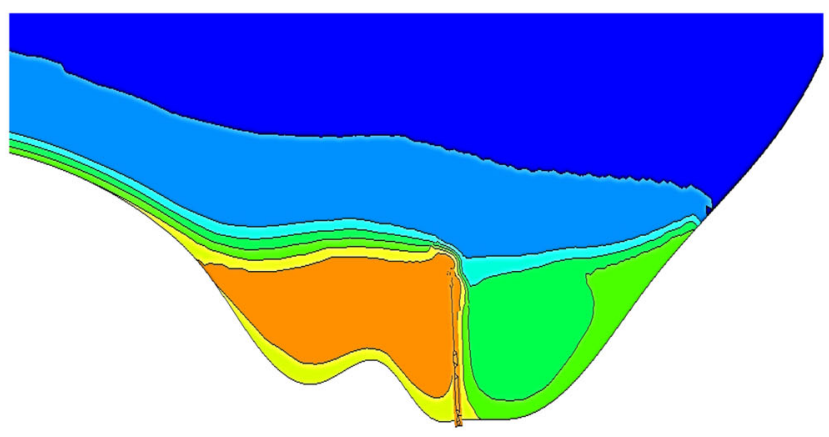

$301.15 \quad 302.15 \quad 303.15 \quad 304.15305 .15306 .15 \quad 307.15$

(a)

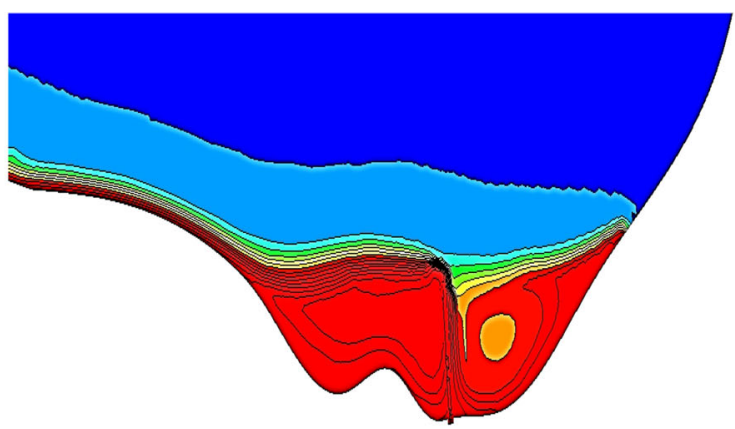

$\alpha$

301.15302 .15303 .15304 .15305 .15306 .15307 .15308 .15309 .15310 .15311 .15312 .15313 .15314 .15315 .15316 .15

(b)

Figure 20. Effect of increase in in blow down temperature (a) blowdown temperature is $8 \mathrm{~K}$ and (b) $16 \mathrm{~K}$ above the ambient water temperature.

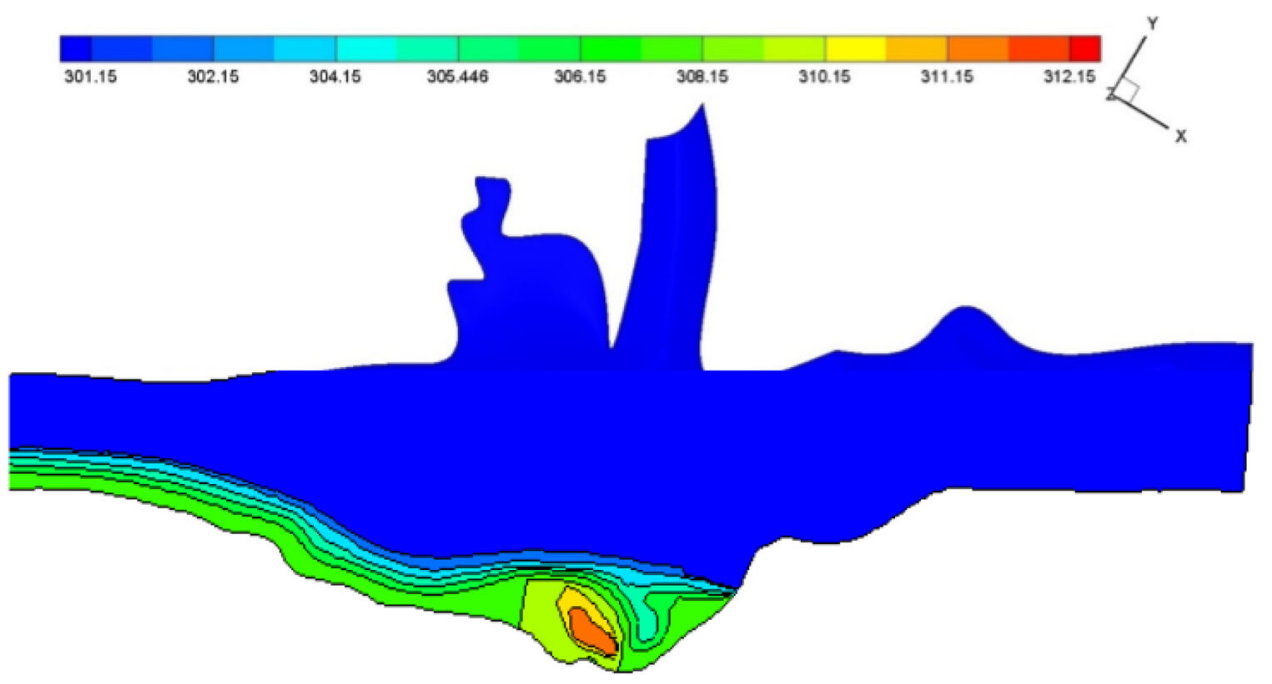

Figure 21. Temperature contours (in K) for wind speed of $3 \mathrm{~m} / \mathrm{s}$ tangential to the direction of Lake flow.

It can be observed from figure 23(a) that due to wind flow the mixing zone is enlarged but is still lesser than those obtained from experimental measurements shown in figure 23(b). The zone of influence reported in figure 23(a) corresponds to the maximum possible wind speed of $3 \mathrm{~m} / \mathrm{s}$ [27]. It is to be noted here that measured 


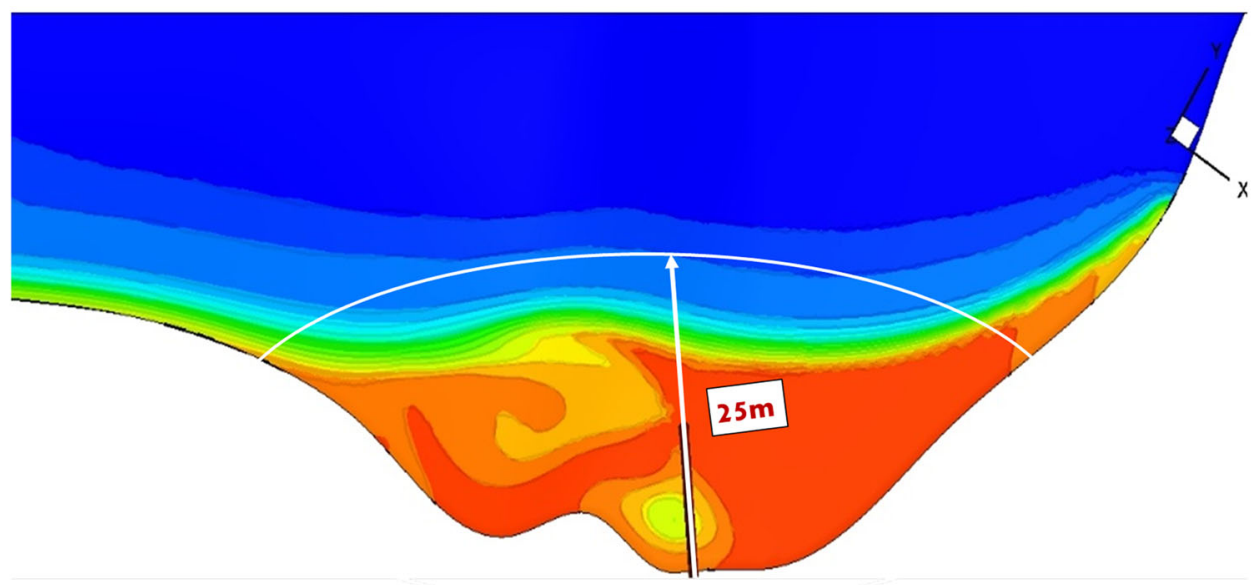

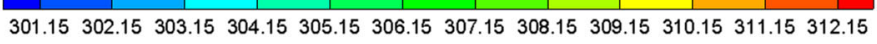

Figure 22. Numerically estimated zone of influence near the blowdown for no wind speed.

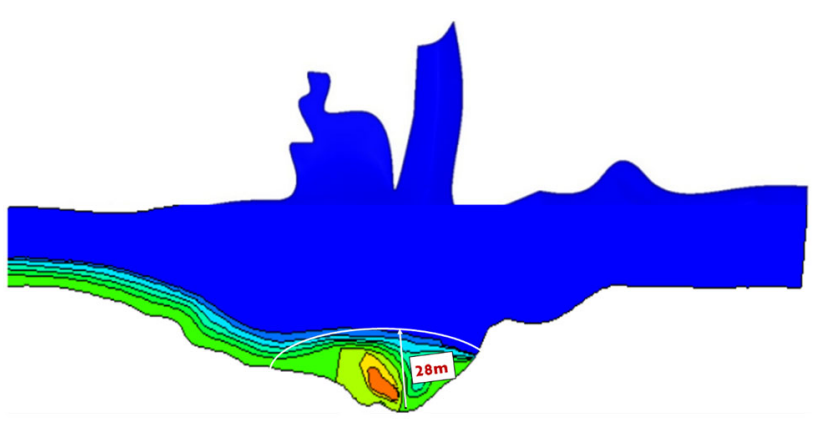

(a)

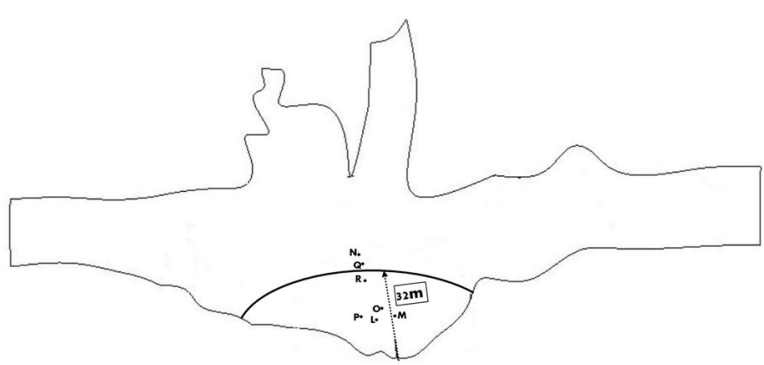

(b)

Figure 23. Zone of influence estimated by (a) numerical simulation for wind speed of $3 \mathrm{~m} / \mathrm{s}$ and (b) field measurement.

data tabulated in table 1 shows wind speeds ranging between $0.6 \mathrm{~m} / \mathrm{s}$ to $1.7 \mathrm{~m} / \mathrm{s}$. Experimental measurements show that the mixing zone extends up to point $\mathrm{R}$ (figure 5) which is higher than the numerical observations. This difference can be attributed to several factors including the presence of weeds and water hyacinth in the lake.

\section{Observations and conclusion}

Major observations drawn from the present work are:

1. A numerical simulation carried out for thermal dispersion of effluent released from the plant into the lake establishes the zone of influence to be up to a distance of $28 \mathrm{~m}$ normal to the mainstream flow when a maximum possible wind speed of $3 \mathrm{~m} / \mathrm{s}$ is considered in the simulations.

2. The increase in secondary inlet flow from inlet II results in a decrease in zone of influence and accumulation of heated water in a smaller zone in left bank canal because of the position of inlet II which is exactly opposite to the location of blowdown. This means that the aquatic habitat near the left bank canal will be significantly affected by the thermal shock. The increase in flow rate of inlet II happens when irrigation department releases more water.

3. The simulation results show significant effect on the zone of influence when wind velocity was accounted for. The wind velocity can reach to the order $3 \mathrm{~m} / \mathrm{s}$ near the blowdown. The zone of influence established based on numerical simulations incorporating the wind speed shows better match with the field measurements. The difference is because of the presence of floating water hyacinth present in the lake body which act as insulators thereby increasing the effective domain of dispersion.

In conclusion, it is established that the position of the blowdown, discharge methodology adopted at blowdown in the existing plant and the location of inlet-II (which is the main source of ambient water flow) minimizes the zone of influence in the power plant considered for the present case study. 


\section{Acknowledgement}

The authors gratefully acknowledge the financial support extended by Board of Research in Nuclear Sciences (BRNS), DAE, Govt. of India, Ref: No. 36(4)/14/22/ 2014-BRNS/1143 dated 04-08-2014 for this research.

\section{Nonmenclature}

$U$ velocity $(\mathrm{m} / \mathrm{s})$

$L$ length (m)

$Q$ discharge $\left(\mathrm{m}^{3} / \mathrm{s}\right)$

$g$ acceleration due to gravity, $9.81 \mathrm{~m} / \mathrm{s}^{2}$

$D$ hydraulic diameter $(\mathrm{m})$

$v$ kinematic viscosity $\left(\mathrm{m}^{2} / \mathrm{s}\right)$

$H$ water depth (m)

$\rho$ density $\left(\mathrm{kg} / \mathrm{m}^{3}\right)$

\section{Subscripts \\ $M$ model \\ $P$ prototype}

\section{References}

[1] Kirillin G, Shatwell T and Kasprzak P 2013 Consequences of thermal pollution from a nuclear plant on lake temperature and mixing regime. J. Hydrol. 496 47-56

[2] Kailasam M 2004 Effect of thermal effluent discharge on benthic fauna off Tuticorin bay south east coast of India. Indian J. Mar. Sci. 33(2): 194-201

[3] Foley B, Jones I D, Maberly S C and Rippey B 2012 Longterm changes in oxygen depletion in a small temperate lake: effects of climate change and eutrophication. Freshw. Biol. 57: 278-289

[4] Abo El-Maaty D A and Hamza R S 2012 Primary amoebic meningoencephalitis caused by Naegleria Fowleri. PUJ. 5: 93-104

[5] El-Alfy K S 2004 Surface discharges of warm water from thermal power stations into rivers. In: 8th International Water Technology Conference, Alexandria, 26-28

[6] Rosen M A, Bulucea C A, Mastorakis N A, Bulucea C A, Jeles C A and Brindusa C C 2015 Evaluating the thermal pollution caused by wastewaters discharged from a chain of coal-fired power plants along a river. Sustainability 7: 5920-5943

[7] El-Ghorab E A S 2013 Physical model to investigate the effect of the thermal discharge on the mixing zone (Case study: North Giza power plant, Egypt). Alex. Eng. J. 52: 175-185

[8] Chieh S 1985 Two-dimensional numerical model of thermal discharges in coastal regions. J. Hydraul. Eng. 113(8): 1032-1040

[9] Zacharias I and Ferentino G 1997 A numerical model for the winter circulation in Lake Trichonis, Greece. Environ. Model. Softw. 12(4): 311-321

[10] Yamanaka R, Nakatsuji K, Liang S and Inoue Y 2005 Effects of thermal stratification and wind blowing on hydrodynamics in the Bohai Sea with notably shallow depths. In:
Proceedings of ninth international conference on estuarine and coastal modeling

[11] Zeng P, Chen H, Ao B, Ji P, Wang X and Ou Z 2002 Transport of waste heat from a nuclear power plant into coastal water. Coast. Eng. 44: 301-319

[12] Fan S, Feng M and Liu Z 2009 Simulation of water temperature distribution in Fenhe Reservoir, Water Sci. Eng. 2(2): 32-42

[13] Shaikh M A 2015 Thermal dispersion model for cooling water of thermal power plant system. Int. J. Curr. Eng. Technol. 5(4): 2472-2477

[14] Abbaspour M, Javid A H, Moghimi P and Kayhan K 2005 Modeling of thermal pollution in coastal area and its economical and environmental assessment. Int. J. Environ. Sci. Tech. 2: 13-26

[15] Lowe S A, Schuepfer F and Dunning D J 2009 Case study: Three-dimensional hydrodynamic model of a power plant thermal discharge. J. Hydraul. Eng. 135(4): 247-256

[16] Liu W, Chen W and Chiu C 2012 Numerical modeling of hydrodynamic and hydrothermal characteristics in subtropical alpine lake. Appl. Math. Model. 36: 2094-2109

[17] Ahmed Z M M, Mehboob-ul-Kabir M and Abdul Hye JM 2001 Modelling of heat dispersion of hot water discharge of the cooling plant of the Meghnaghat power plant. Int. Congr. Model. Simul. Proc. 2(2): 257-263

[18] Demenet P and Bae B K 2014 Shoaiba II power plant hydraulic studies. In: Proceedings of 34th Conference on Coastal Engineering

[19] Prodanovic P 2013 Hydrodynamic and thermal plume modeling for waste heat discharges into coastal waters. In: 4th Specialty Conference on Coastal, Estuary and Offshore Engineering

[20] Dargahi B and Setegn S G 2011 Combined 3D hydrodynamic and watershed modelling of Lake Tana, Ethiopia $J$. Hydrol. 398: 44-64

[21] Sun X, Bian H, Luo C, Li J and Wang L 2011 Numerical simulation of seasonal variation of temperature in the Bohai Sea. Procedia Environ. Sci. 10: 1914-1919

[22] Yasser M S, Mohamed B E and Mohamed M A 2014 Power plant intakes performance in low flow water bodies. Water Sci. 29: 54-67

[23] Saptarini D and Fudlailah P 2015 On defining the effects of water temperatures increase to the coral reef: a case study of cooling water discharge from a power generation. Procedia Earth Planet. Sci. 14: 152-160

[24] Fossati M, Santoro P, Urrestarazu S and Piedra-Cueva I 2011 Numerical study of the effect of a power plant cooling water discharge in the Montevideo Bay, Research Article. J. Appl. Math. Article ID 970467, 23 pages

[25] German A, Espino M, Blasco J and Maidana M 2007 Finite element modelling of the thermal outflow of three power plants in Huelva Estuary. Adv. Eng. Softw. 38: 379-385

[26] Sasmal K, Bonthu S R, Maity S and Warrior H V 2015 Effect of atmospheric forcing on plume dispersion and study of nuclear effluent trajectories for the Kalpakkam Coast, India J. Hazard. Toxic Radioact. Waste 19(3): 04014035

[27] Prasad Y S R 1989 Environmental appraisal for proposed Kakrapar Site

[28] Dost R J J and Mannaerts C M M 2008 Generation of Lake Bathymetry using sonar, satellite imagery and GIS. In: Proceedings of the 2008 ESRI international user conference 